\title{
DIFFERENTIALS ON QUOTIENTS OF ALGEBRAIC VARIETIES
}

\author{
BY
}

\author{
CAROL M. KNIGHTEN
}

\begin{abstract}
The relations between differentials invariant with respect to a finite group acting on a variety and the differentials on the quotient variety are studied. If the quotient map is unramified in codimension 1 we have an isomorphism for Zariski differentials, but not in general for Kähler differentials. Necessary and sufficient conditions for isomorphism of the Zariski differentials are given when the finite group acts linearly. Examples illustrate the scope of the theorems and some open problems.
\end{abstract}

Introduction. This paper is devoted to discussing differentials on quotients of algebraic varieties by finite group actions. If $X$ is a quasi-projective variety over a field $k$ and $G$ is a finite group of $k$-automorphisms of $X$, then the orbit space $X / G$ is a quasi-projective variety and the quotient map $\pi: X \rightarrow X / G$ is integral and of finite type. Varieties of the form $X / G$ have been frequently though not systematically discussed in the literature. For example, see Serre's Groupes algébriques [9, III.12]. Of particular interest are symmetric products of a variety $Y$ (here $X=Y^{r}$ and $G$ is the $r$-fold symmetric group acting by permuting the factors), which have been discussed, for example, by Mattuck ([5][7]). Moreover Briney [1] has shown that on varieties of the form $X / G$ with $X$ nonsingular there is a "good" intersection theory with rational coefficients.

In connection with both symmetric products and intersection theory it is desirable to have detailed information about the relation between differentials on $X / G$ and invariant differentials on $X$. Some information in that direction is obtained here.

We begin, in Chapter I, with some elementary properties of Zariski differentials. There are two novelties-we introduce and work with the sheaf of Zariski differentials, and we prove the (well-known?) result that the sheaf of Zariski differentials is the double dual of the sheaf of Kähler differentials.

Chapter II is devoted to showing that if the quotient map is tamely ramified in codimension 1 , then the module of $G$-invariant Zariski differentials on $X$ is isomorphic to the module of Zariski differentials on $X / G$. This, combined with other results about Zariski differentials, allows us to give examples showing that no such results hold for Kähler differentials.

Received by the editors October 25, 1971.

AMS (MOS) subject classifications (1970). Primary $14 \mathrm{~F} 10$.

Key words and phrases. Quotient of algebraic variety, Zariski differentials, Kähler differentials, invariant differentials, ramified, action of finite group.

Copyright $\odot 1973$, American Mathematical Society 
In Chapter III we give necessary and sufficient conditions for the module of Zariski differentials on $X / G$ to be isomorphic to the module of invariant Zariski differentials on $X$ when $G$ acts linearly on the nonsingular variety $X$. ( $G$ acts linearly on $X$ if at every closed point the action of the inertia group of that point on the completion of its local ring is linear.) The proof is by reduction to affine space followed by detailed computations.

Chapter IV consists of examples to illustrate the significance of the theorem of Chapter III together with other examples which indicate the difficulties of the nonlinear case.

The main results of Chapters II-IV require the assumption that the base field $k$ is algebraically closed.

Parts of this paper-especially a special case of the theorem of Chapter IIIformed the principal content of the author's doctoral thesis which was submitted to the Massachusetts Institute of Technology in August 1967. Special thanks must go to my the sis advisor, Professor Arthur Mattuck, for his advice and criticism while I was a student, and also for his insistence that I extend the thesis to the full power and generality obtained here.

My deepest appreciation is reserved for my husband, Bob, whose patience and encouragement made this paper possible, and who spent untold hours rewriting it in a form others could read.

\section{CHAPTER I. GENERALITIES A BOUT DIFFERENTIALS}

In this chapter we generalize some definitions and results of Zariski [11, $\S 8$ ] on differentials to the context of sheaves and prove that the sheaf of Zariski differentials is the double dual of the Kähler sheaf of differentials. This last seems to be well known, but we know of no proof in the literature.

Throughout this chapter $\left(X, \mathcal{O}_{X}\right)$ will denote a variety over the field $k$. The definitions given here agree with those of Zariski [op. cit.] for $\left(X, \mathcal{O}_{X}\right)$ a normal variety over an algebraically closed field $k$.

Let $K$ be the field of rational functions on $\left(X, \mathcal{O}_{X}\right)$. Note that $K$ may be considered a constant sheaf on $X$, and $\mathcal{O}_{X}$ is a subsheaf of $K$.

Definition. $\mathfrak{D}_{K}=\operatorname{Der}_{k}(K, K)$ is the $K$ vector space of $k$-derivations of $K$.

Definition. $\mathscr{D}_{X}$ is the presheaf on $X$ defined by $\mathscr{D}_{X}(U)=\left\{D \in \mathfrak{D}_{K} \mid\right.$ for all $x$ in $\left.U, D\left(\mathcal{\Theta}_{x}\right) \subseteq \mathcal{\Theta}_{x}\right\}$, with the obvious restriction maps.

Notice that if $U$ is open in $X$, then $\mathcal{O}_{U}=\left.\mathcal{O}_{X}\right|_{U}$ may be considered as a subsheaf of $K$ considered as a constant presheaf on $U$. Thus if $D \in \mathscr{D}_{K}$, then $D\left(\Theta_{U}\right)$ is a subsheaf of $K$, and we can ask when it is a subsheaf of $\mathcal{O}_{U}{ }^{\circ}$.

Lemma 1. $\mathscr{D}_{X}(U)=\left\{D \in \mathscr{D}_{K} \mid D\left(\mathcal{O}_{U}\right) \subseteq \mathcal{O}_{U}\right.$ where $D$ is considered as a map of sheaves\}. 
Proof. For $V$. open in $U, \mathcal{O}_{U}(V) \subseteq \mathcal{O}_{x}$ for all $x$ in $V$. Thus if $D \in \mathscr{D}_{X}(U)$, then $D\left(\Theta_{U}(V)\right) \subseteq D\left(\Theta_{x}\right) \subseteq \Theta_{x}$ for all $x$ in $V$, i.e., $D\left(\Theta_{U}(V)\right) \subseteq \bigcap_{x \in V} \Theta_{x}=$ $\Theta_{U}(V)$. That is $D\left(\Theta_{U}^{x}\right) \subseteq \Theta_{U}^{x}$.

Conversely if $D\left(\mathcal{\Theta}_{U}\right) \subseteq \mathcal{\Theta}_{U}$ then of course $D\left(\mathcal{\Theta}_{x}\right) \subseteq \mathcal{\Theta}_{x}$ for all $x \in U$.

Directly analogous to the $\mathcal{O}_{X}$ module of homomorphisms between two $\mathcal{\theta}_{X}$ modules is the $\mathcal{O}_{X}$ module $\operatorname{Der}_{k}\left(\mathcal{O}_{X}, \pi\right)$ of derivations of $\mathcal{O}_{X}$ into an $\mathcal{O}_{X}$ module $\mathbb{M}$. Moreover if $\mathbb{M}$ is quasi-coherent, then $\operatorname{Der}_{k}\left(\Theta_{X}, \mathbb{M}\right)$ is quasi-coherent. In particular if $X=\operatorname{Spec} A$ and $\mathbb{M}=M^{\sim}$ then $\operatorname{Den}_{k}\left(\Theta_{X}, \mathbb{M}\right)=\operatorname{Der}_{k}(A, M)^{\sim}$. For details see [3, EGA IV 16.5.6, 16.5.2].

Lemma 2.

$$
\begin{aligned}
\mathscr{D}_{X}(U) & \longrightarrow \operatorname{Der}_{k}\left(\Theta_{U}, \Theta_{U}\right) \\
D & \left.\longmapsto D\right|_{\vartheta_{U}}
\end{aligned}
$$

is an isomorphism.

Thus the presheaf $\mathfrak{D}_{X}$ is isomorpbic to the quasi-coberent sheaf $\operatorname{Der}_{k}\left(\Theta_{X}, \Theta_{X}\right)$ and bence is a quasi-coberent sheaf.

Proof. Let $D \in \operatorname{Der}_{k}\left(\Theta_{U}, \Theta_{U}\right)$ and let Spec $A$ be any affine open subvariety of $U$; then $D$ gives a $k$-derivation of $A$. Now since $K$ is the quotient field of $A$ this derivation extends uniquely to a $k$-derivation $D^{A}$ of $K$. We first show that $D^{A}$ is independent of $A$. Namely if Spec $B$ is also an open affine set in $U$, then Spec $A \cap \operatorname{Spec} B$ is an affine open set with an affine ring $C$ containing both $A$ and $B$. Now since all are induced by $D,\left.D^{C}\right|_{A}=\left.D^{A}\right|_{A}$ and $\left.D^{C}\right|_{B}=$ $\left.D^{B}\right|_{B}$, so $D^{A}=D^{C}=D^{B}$. Denote this unique derivation of $K$ gotten from $D$ by $D^{\prime}$.

To show that $\left.D^{\prime}\right|_{\theta_{U}}=D$.it suffices to show that for $x \in U,\left.D^{\prime}\right|_{\theta_{x}}=D_{x}$ (the derivation of $\mathcal{O}_{x}$ induced by $D$ ). $x$ is in some affine open subset Spec $A$ of $U$ and $\mathcal{O}_{x}$ can be identified with $A_{\mathfrak{p}}$ where $\mathfrak{P}$ is the prime ideal corresponding to $x ;\left.D^{\prime}\right|_{A_{p}}=D_{x}$ since $\left.D^{\prime}\right|_{A}=\left.D_{x}\right|_{A}$.

Proposition 1. $\mathscr{D}_{X}$ is a quasi-coberent sbeaf. If $U=\operatorname{Spec} A$ is an affine open set in $X$, then $\left.D_{X}\right|_{U} \simeq \mathscr{D}_{A} \sim$ where $\mathscr{D}_{A}=\left\{D \in \mathscr{D}_{K} \mid D(A) \subseteq A\right\} \cong \operatorname{Der}_{k}(A, A)$.

Proof. This follows from Lemma 2 and [3, EGA IV 16.5.2].

Proposition 2. The stalk of $\mathfrak{D}_{X}$ at $x$ is $\mathscr{D}_{x}=\left\{D \mid D \in \mathscr{D}_{K}, D\left(\mathcal{O}_{x}\right) \subseteq \mathcal{O}_{x}\right\}$.

Proof. A typical element of $\mathscr{D}_{x}$ is the germ of $D_{1} \in \mathscr{D}_{U_{1}}$ where $U_{1}$ is an open neighborhood of $x$.

If $D_{2} \in \mathscr{I}_{U_{2}}$ defines the same element of $\mathscr{D}_{x}$ as does $D_{1}$, then of course $\left.D_{1}\right|_{\mathcal{O}_{x}}=\left.D_{2}\right|_{\mathcal{O}_{x}}$ and so we have a homomorphism $\mathscr{D}_{x} \rightarrow\left\{D \mid D \in \mathscr{D}_{k}, D\left(\mathcal{O}_{x}\right) \subseteq \mathcal{O}_{x}\right\}$. 
There is also the obvious isomorphism

$$
\left\{D \mid D \in \mathscr{D}_{K}, D\left(\mathcal{O}_{x}\right) \subseteq \mathcal{O}_{x}\right\} \longrightarrow \operatorname{Der}_{k}\left(\Theta_{x}, \Theta_{x}\right) .
$$

Now given $D \in \operatorname{Der}_{k}\left(\Theta_{x}, \Theta_{x}\right)$ we can find an affine open subset Spec $A$ of $X$ containing $x$ so that $D$ becomes an element of $\operatorname{Der}_{k}\left(A_{\mathfrak{p}}, A_{\mathfrak{p}}\right), \mathfrak{p}$ being the prime ideal of $x$. Let $a_{1}, \cdots, a_{r}$ be generators of $A$, write $D\left(a_{i}\right)=b_{i} / c_{i}$ with $c_{i} \in A-\not$ and put $f=c_{1} \cdots c_{r}$. Since $D$ is a derivation $D\left(A_{f}\right) \subseteq A_{f}$. Moreover $x \in \operatorname{Spec} A_{f} \subseteq X$ since $f \notin$ fo. $D$ extends uniquely to a derivation $D^{\prime}$ in $\mathscr{D}_{X}\left(\operatorname{Spec} A_{f}\right)$ and so defines an element of $\mathscr{D}_{x}$. This gives a homomorphism $\operatorname{Der}_{k}\left(\mathcal{O}_{x}, \mathcal{O}_{x}\right) \rightarrow D_{x}$ which is clearly the inverse of the above map.

Definition. $\Omega^{z}(K)=\operatorname{Hom}_{K}\left(\mathscr{D}_{K}, K\right)$.

Definition. $\Omega^{z}{ }_{X}$ is the presheaf on $X$ defined by $\Omega^{z}{ }_{X}(U)=\left\{\omega \in \Omega^{z}(K) \mid \forall x \in\right.$ $\left.U, \forall D \in \mathscr{D}_{x}, \omega(D) \in \mathcal{O}_{x}\right\}=\left\{\omega \in \Omega^{z}(K) \mid \forall x \in U, \omega\left(\mathscr{D}_{x}\right) \subseteq \mathcal{O}_{x}\right\}$. Note that $\mathscr{D}_{x \mid U}$ is $\mathscr{D}_{U}$ since $X$ and $U$ have the same function field. $\Omega^{z}{ }_{X}$ is called the sheaf of Zariski defferentials on $X$.

Lemma 3. For $U=\operatorname{Spec} A$ affine open in $X$ and $D \in D_{K}$ there exists $0 \neq$ $c \in K$ such that $c D \in \mathscr{D}_{X}(U)$, that is, $c D\left(\mathcal{O}_{x}\right) \subseteq \mathcal{O}_{x}$ for all $x \in U$.

Proof. Let $a_{1}, \cdots, a_{r}$ be a finite set of generators of $A$ over $k$. Then $D\left(a_{i}\right)=b_{i} / c_{i}$ for some $b_{i}, c_{i}$ in $A$. Let $c=c_{1} \cdots c_{r}$ so $c D(A) \subseteq A$. But then $c D\left(A_{\mathfrak{p}}\right) \subseteq A_{\mathfrak{p}}$ for $\mathfrak{D}$ any prime ideal of $A$, i.e., $c D\left(\Theta_{x}\right) \subseteq \mathcal{O}_{x}$ for any $x \in U$.

Lemma 4.

$$
\begin{aligned}
\Omega_{X}^{z}(U) & \longrightarrow \operatorname{Hom}_{\mathcal{E}_{U}}\left(\mathfrak{D}_{U}, \mathcal{O}_{U}\right) \\
\omega & \left.\longmapsto\right|_{\mid \mathcal{D}_{U}}
\end{aligned}
$$

is an isomorphism for $U$ affine open in $X$.

Proof. Define $f_{U}: \mathfrak{D}_{X}(U) \otimes_{\mathcal{O}_{X}(U)} K \rightarrow \mathfrak{D}_{K}$ by $f_{U}(D \otimes a)=a D$. Then $f_{U}$ is an isomorphism with inverse $f_{U}^{-1}$ where $f_{U}^{-1}(D)=a D \otimes 1 / a$ where $a$ is any nonzero element of $K$ such that $a D \in \mathscr{D}_{X}(U)$. Such an $a$ exists by the last lemma.

Now given $g: \mathscr{D}_{U} \rightarrow \mathcal{O}_{U}$ we have $g \otimes_{\mathcal{O}_{U}} K: \mathscr{D}_{K} \rightarrow K$ and this composed with the inclusion $\mathscr{D}_{U} \rightarrow \mathfrak{D}_{K}$ gives an element of $\Omega^{z}{ }_{X}(U)$. It is easily checked that this is the inverse of $\left.\omega \mapsto \omega\right|_{\mathbb{D}_{U}}$.

Corollary 1. $\Omega^{z}{ }_{X} \rightarrow \mathcal{H}_{a m} \mathcal{O}_{X}\left(\mathfrak{D}_{X}, \mathcal{O}_{X}\right)$ is an isomorphism.

For any $\mathcal{O}_{X}$ module $\mathcal{F}$ we will write $\mathcal{F}^{*}$ for the dual module Hom $\mathcal{O}_{X}\left(\mathcal{F}, \mathcal{O}_{X}\right)$. With this notation Corollary 1 says that $\Omega^{z}{ }_{X}$ is canonically isomorphic to $\mathfrak{D}_{X}{ }^{*}$. 
Corollary 2. $\Omega_{x}^{z}=\left\{\omega \in \Omega^{z}{ }_{K} \mid \omega\left(\mathscr{D}_{x}\right) \subseteq \mathcal{O}_{x}\right\}$.

The defining property of the Kähler module of differentials on $X$ is that there is a derivation $d: \mathcal{O}_{X} \rightarrow \Omega_{X}$ so that

$$
\begin{aligned}
\operatorname{Hom}_{X}\left(\Omega_{X}, \mathfrak{F}\right) & \longrightarrow \operatorname{Der}_{k}\left(\mathcal{O}_{X}, \mathfrak{F}\right) \\
f & \longmapsto d d
\end{aligned}
$$

is an isomorphism for every quasi-coherent $\mathcal{O}_{X}$ module $\mathcal{F} . \Omega_{X}$ is itself a quasicoherent $\Theta_{X}$ module (see [3, EGA, IV 16.5.6]).

Corollary 3. $H: \Omega_{X}^{z} \rightarrow \Omega_{X}{ }^{* *}$ is an isomorphism where $H$ is defined by $H_{U}(\omega)=\omega^{* *}$ and $\omega^{* *}(f)=\omega(f d)$.

Note that this makes sense, for if $f \in \Omega_{X}{ }^{*}$, i.e., $f \in \operatorname{Hom}_{\Theta_{U}}\left(\Omega_{U}, \mathcal{O}_{U}\right)$, then $f d \in \operatorname{Der}_{k}\left(\mathcal{O}_{U}, \mathcal{O}_{U}\right)$ which is identified, via Lemma 2, with $\mathscr{D}_{X}(U) \subseteq \mathscr{D}_{K}$ and so $f d$ is in the domain of $\omega$.

The basic property of Zariski differentials, which is used throughout the rest of this work, is contained in

Theorem 1. Let $X$ be a normal variety. For any point $x_{0}$ of $X$, if a Zariski differential $\omega$ is in $\Omega^{z}{ }_{x}$ for every point $x$ of codimension 1 passing through $x_{0}$, then $\omega \in \Omega_{x_{0}}^{z}$.

Proof. We want to prove that $\Omega_{x_{0}}^{z}=\bigcap \Omega_{x}^{z}$ where the $x$ are as in the statement of the theorem. But this is the same as showing that $\Omega^{z} x_{0}=\bigcap\left(\Omega_{x_{0}}^{z}\right)_{p}$ where $p$ ranges over all minimal primes of $\mathcal{O}_{x_{0}}$. Now $\Omega_{x_{0}}^{z}$ is reflexive because $\Omega_{x_{0}}^{z}=\Omega_{x_{0}}{ }^{* *}$, and the theorem follows from the classical result that a torsion free finitely generated module $M$ over an integrally closed Noetherian domain $A$ is reflexive if and only if $M=\bigcap M_{p}$ as $b$ ranges over the minimal prime ideals of $A$ (see Samuel [8]).

Corollary 4. Let $X$ be as above. If $\omega \in \Omega^{z}(K), U$ is open in $X$ and $\omega$ is in $\Omega^{z}{ }_{x}$ for every $x$ of codimension 1 in $U$, then $\omega \in \Omega^{z}{ }_{X}(U)$.

Definition. $d^{z}: \mathcal{\Theta}_{X} \rightarrow \Omega^{z}{ }_{X}$ is defined, on sections over $U$, by $d^{z}{ }_{U}(a)(D)=$ $D(a)$. Here $\Gamma\left(U, \Omega^{z}{ }_{X}\right)$ is identified with $\mathscr{D}_{X}(U)^{*}$ via Corollary 1 . Note that $d^{z}$ is a derivation.

Proposition 3. The diagram

commutes.

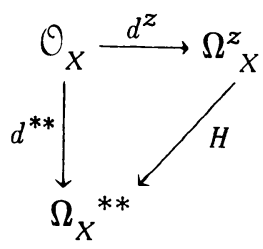


Proof. For $a \in \mathcal{O}_{X}(U)$ and $f \in \Omega_{X}{ }^{*}(U)$ we have $H\left(d^{z}(a)\right)(f)=d^{z}(a)(f \circ d)=$ $f(d(a))=d^{* *}(a)(f)$.

Let $e: \Omega_{X} \rightarrow \Omega_{X}{ }^{* *}$ be the canonical homomorphism and $e^{\prime}: \Omega_{X} \rightarrow \Omega_{X}^{z}$ the unique homomorphism such that $e^{\prime} \circ d_{X}=d^{z}{ }_{X}$. Then from Corollary 3 and the last proposition it follows that the diagram

commutes.

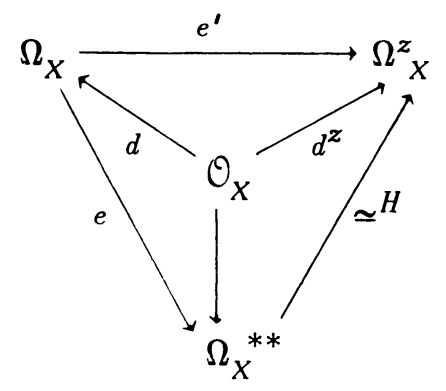

From the above diagram we note that $\operatorname{Ker} e^{\prime}=\operatorname{Ker} e$. But $\operatorname{Ker} e=t \Omega_{X}$ (the torsion submodule of $\Omega_{X}$ ). In fact more generally we quote the well-known

Proposition 4. If $R$ is a Noetherian domain and $M$ is a finitely generated $R$ module, then the kernel of the canonical homomorphism $e: M \rightarrow M^{* *}$ is $t M$, the torsion submodule of $M[4, p .897]$.

In the next chapter we will obtain conditions enabling us to compute $\Omega^{z}{ }_{X / G}$ in certain cases. If $e$ were an isomorphism or even an epimorphism we would have somewhat similar results for $\Omega_{X / G}$. Unfortunately $e$ is in general neither monic nor epic.

An example where $e$ is monic but not epic is given by the cone $X=$ Spec $k[X, Y, Z] /\left(X Y-Z^{2}\right)$. If $X=\operatorname{Spec}\left(k[X, Y, Z, W] /\left(X W-Y Z, X Z-Y^{2}, Y W-Z^{2}\right)\right)$, the cone over the twisted cubic, then $e$ is neither a monomorphism nor an epimorphism. I have been unable to find an example where $e$ is epic but not monic. Of course if $X$ is nonsingular $e$ is an isomorphism, but I know of no other cases where this is so.

\section{CHAPTER II. THE TAMELY RAMIFIED CASE}

The notation here is as in Chapter I except that if confusion is likely we write $K(X)$, rather than $K$, for the field of rational functions on $X$.

From Theorem 2 (in $\$ 2$ ) onwards the base field $k$ is algebraically closed.

1. Invariant differentials. For $f: X \rightarrow Y$ a dominant, integral morphism of normal varieties we define a map $\Omega^{z} f: \Omega^{z}{ }_{Y} \rightarrow f_{*} \Omega^{z}{ }_{X}$, of $\Theta_{Y}$-modules as follows: Since $f$ is dominant we have the inclusion $f^{*}: K(Y) \rightarrow K(X)$, and, since the image of the respective $d^{z}$ 's generate $\Omega^{z}(K(Y))$ and $\Omega^{z}(K(X))$, there is a 
unique $K(Y)$ homomorphism $\Omega^{z} f: \Omega^{z}(K(Y)) \rightarrow \Omega^{z}(K(X))$ so that $\Omega^{z} f d^{z}=d^{z} f^{*}$. Notice that $\Omega^{z} f$ is given explicitly by $\Omega^{z} f(\omega)(D)=\omega\left(D f^{*}\right)$ for $\omega \in \Omega^{z}(K(Y))$ and $D \in \mathscr{D}_{K(X)}$, and the force of the above remark is that this formula makes sense, i.e., $D f^{*}$ is in the domain of $\omega$. Finally we define $\Omega^{z} f: \Omega^{z}{ }_{Y}(U) \rightarrow f_{*} \Omega^{z}{ }_{X}(U)$ by $\Omega^{z} f(\omega)(D)=\omega\left(D f^{*}\right)$ for $\omega \in \Omega^{z}{ }_{Y}(U)$ and $D \in D_{X}\left(f^{-1}(U)\right)$. By the above remark we know that $\Omega^{z} f(\omega) \in \Omega^{z}(K(X))$, but we must check that it is in $f_{*} \Omega^{z}{ }_{X}(U)=$ $\Omega_{X}^{z}\left(f^{-1}(U)\right)$.

Now from the corollary to Theorem 1 of Chapter $I \Omega^{z}{ }_{Y}(U)$ is the intersection of all $\Omega_{X, y}^{z}$ with $y$ a point of codimension 1 in $U$. Similarly $\Omega_{X}^{z}\left(f^{-1}(U)\right)$ is the intersection of all $\Omega_{X, x}^{z}$ for $x$ a point of codimension 1 in $f^{-1}(U)$. Since $f$ is integral there is for each $y$ of codimension 1 in $U$ an $x$ of codimension 1 in $f^{-1}(U)$ with $f(x)=y$. Thus it suffices to show that $\Omega^{z} f\left(\Omega^{z}{ }_{Y, y}\right) \subseteq\left(f_{*} \Omega^{z}{ }_{X}\right)_{y}=$ $\bigcap_{f(x)=y} \Omega_{X, x}^{z}$ for each such $y$. But

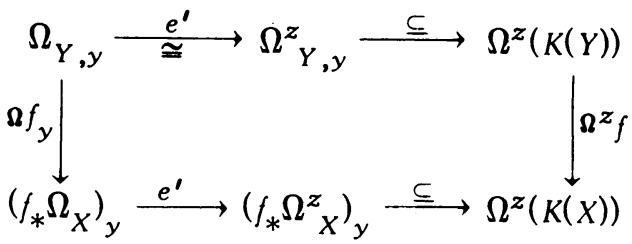

is commutative and, $y$, being a point of codimension 1 on a normal variety, $\Omega_{Y, y}$ is a free module of finite rank and so $e^{\prime}$ is an isomorphism as indicated in the diagram. Thus $\Omega^{z} f\left(\Omega_{Y, y}^{z}\right) \subseteq\left(f_{*} \Omega^{z}{ }_{X}\right)_{y}$ as needed.

Now let $X$ be a normal, quasi-projective $k$-variety and $G$ a finite group of $k$-automorphisms of $X$. Then there is a normal, quasi-projective quotient variety $X / G$ and the quotient map $\pi: X \rightarrow X / G$ is a dominant, integral morphism. For details see [2, SGA 1, Exp. V].

$\pi$ being a dominant, integral morphism we have the map $\Omega^{z} \pi: \Omega_{X / G}^{z} \rightarrow$ $\pi_{*} \Omega^{z}{ }_{X}$. As expected this factors through $\left(\pi_{*} \Omega^{z}{ }_{X}\right)^{G}$ as we will see as soon as we define it.

$\sigma \in G$ gives $\Omega^{z} \sigma: \Omega^{z}{ }_{X} \rightarrow \sigma_{*} \Omega^{z}{ }_{X}$ and applying $\pi_{*}$ to this gives $\pi_{*} \Omega^{z} \sigma:$ $\pi_{*} \Omega^{z}{ }_{X} \rightarrow \pi_{*} \sigma_{*} \Omega^{z}{ }_{X}=\pi_{*} \Omega^{z}{ }_{X}$ and this defines an action of $G$ on $\pi_{*} \Omega^{z}{ }_{X}$. For each open set $U$ in $X / G$, we note that $\pi_{*} \Omega^{z}{ }_{X}(U)$ is a subset of $\Omega^{z}(K)$ and the action of $G$ defined above is the restriction of the action on $\Omega^{z}(K)$ defined by $\sigma \cdot \omega(D)=\omega\left(D \sigma^{*}\right)$ for $\sigma \in G, \omega \in \Omega^{z}(K)$ and $D \in \mathscr{D}_{K}$. From this we get the presheaf $\left(\pi_{*} \Omega^{z}{ }_{X}\right)^{G}$ where $\Gamma\left(U,\left(\pi_{*} \Omega^{z}{ }_{X}\right)^{G}\right)=\Gamma\left(U, \pi_{*} \Omega^{z}{ }_{X}\right)^{G}$. This is clearly a sheaf.

Proposition 1. $\Omega^{z} \pi\left(\Omega^{z}{ }_{X / G}\right) \subseteq\left(\pi_{*} \Omega^{z}{ }\right)^{G}$.

Proof. We need only note that $\left(\pi_{*} \Omega^{z} \sigma\right) \Omega^{z} \pi=\Omega^{z} \pi \sigma=\Omega^{z} \pi$ as maps from $\Omega_{X / G}^{z}$ to $\pi_{*} \Omega_{X}^{z}$ 
Remark.

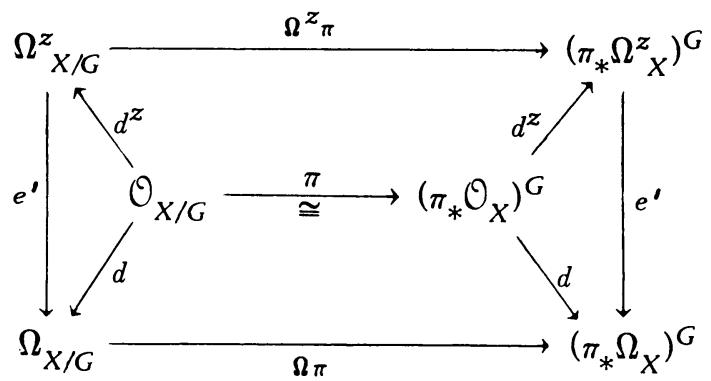

is a commutative diagram.

2. Reduction to consideration of the ramification group. Let $X, G$ and $\pi$ be as above. When is $\Omega^{z} \pi: \Omega_{X / G}^{z} \rightarrow\left(\pi_{*} \Omega_{X}\right)^{G}$ an isomorphism? As a preliminary to answering this question, we prove in this section that for a point $x$ in $X$ with image $y$ in $X / G$ and ramification group $H, \Omega^{z} \pi_{y}$ is an isomorphism iff $\Omega^{z} \rho_{z}$ is an isomorphism where $\rho: X \rightarrow X / H$ is the quotient map and $z=\rho(x)$. Moreover to show $\Omega^{z} \pi$ an isomorphism it is enough to show that $\Omega^{z} \pi_{y}$ is an isomorphism at each point $y$ of codimension 1. The proof of both these assertions is via completions.

For a point $y$ on $X / G,\left(\pi_{*} \Theta_{X}\right)_{y}$ is a semilocal ring on which $G$ acts giving $\mathcal{O}_{X / G, y}$ as ring of invariants. We know that $\Omega^{z} \pi_{y}: \Omega_{X / G, y}^{z} \rightarrow\left(\pi_{*} \Omega^{z}{ }_{X}\right)_{y}{ }^{G}$ is an isomorphism iff its completion is. But $G$ also acts on $\left(\pi_{*} \Omega^{z}{ }_{X}\right)_{y}$ and $\left(\left(\pi_{*} \Omega^{z}{ }_{X}\right)_{y}{ }^{G}\right)^{\wedge} \subseteq\left(\left(\pi_{*} \Omega^{z}{ }_{X}\right)_{y}{ }^{\wedge}\right)^{G}$. It will be helpful to know that the se are equal as the second is easier to handle. Various forms of this situation occur so we formalize it in

Lemma 1. Let $A$ be a semilocal ring, $G$ a finite group acting on $A$ and assume $A^{G}$ is Noetherian. Suppose $G$ acts compatibly on a finitely generated A-module $N$. Then $\left(N^{G}\right)^{\wedge}=\left(N^{\wedge}\right)^{G}$.

Proof. If $x$ is the radical of $A$, then $\Upsilon \cap A^{G}$ is the radical of the semilocal ring $A^{G}$ and the two adic topologies on $N$ are the same. Consider the exact sequence $0 \rightarrow N^{G} \rightarrow N \rightarrow \Pi_{\sigma \epsilon G} N$ of $A^{G}$ modules where $i: N^{G} \rightarrow N$ is the inclusion and $g: N \rightarrow \prod_{\sigma \in G} N$ is defined by $g(n)=(\sigma(n)-n: \sigma \in G)$. Since completion is exact we get the exact sequence $0 \rightarrow\left(N^{G}\right)^{\wedge} \rightarrow N^{\wedge} \rightarrow\left(\Pi_{\sigma \epsilon G} N\right)^{\wedge}$. Moreover $\left(\Pi_{\sigma \epsilon G} N\right)^{\wedge}$ is naturally isomorphic to $\Pi_{\sigma \in G} N^{\wedge}$ and the kernel of the composed map $N^{\wedge} \rightarrow \prod_{\sigma \in G} N^{\wedge}$ is $\left(N^{\wedge}\right)^{G}$, so $\left(N^{G}\right)^{\wedge}=\left(N^{\wedge}\right)^{G}$ as claimed.

Corollary. $\left(A^{\wedge}\right)^{G}=\left(A^{G}\right)^{\wedge}$.

As a first step we reduce the problem to consideration of the decomposition group of a point, i.e., the maximal subgroup of $G$ which leaves the point fixed. 
Theorem 1. Let $X, G$ and $\pi$ be as above. Let $x \in X$, let $H$ be its decomposition group, $\rho: X \rightarrow X / H$ the quotient map and $z=\rho(x), y=\pi(x)$. Then $\Omega^{z} \pi_{y}: \Omega_{X / G, y}^{z} \rightarrow\left(\pi_{*} \Omega^{z}{ }_{X}\right)^{G}$ is an isomorpbism iff $\Omega^{z} \rho_{z}: \Omega_{X / H, z}^{z} \rightarrow\left(\rho_{*} \Omega^{z}{ }_{X}\right)^{H}{ }_{z}$ is an isomorphism.

Proof. As $\pi$ is constant on $H$ orbits there is a unique map $\tau: X / H \rightarrow X / G$ so that $\tau \rho=\pi$. On $X / H$ we have $\Omega^{z} \rho: \Omega_{X / H}^{z} \rightarrow \rho_{*} \Omega^{z}{ }_{X}$. Applying $\tau_{*}$ gives us $\tau_{*} \Omega^{z}(\rho): \tau_{*} \Omega_{X / H}^{z} \rightarrow \pi_{*} \Omega_{X}^{z}$ on $X / G$. Now $\Omega^{z}(\pi)=\tau_{*} \Omega^{z}(\rho) \Omega^{z}(\tau)$, so $\Omega^{z}(\pi)$ factors through $\tau_{*} \Omega^{z}{ }_{X / H}$ as well as through $\left(\pi_{*} \Omega^{z}{ }_{X}\right)^{G}$. We denote the intersection of these two subsheaves of $\pi_{*} \Omega^{z}{ }_{X}$ by $\left(\tau_{*} \Omega^{z}{ }_{X / H}\right)^{G}$. For the remainder of the proof we will write $a$ for $\Omega^{z}(\pi)$ considered as a map into $\left(\tau_{*} \Omega^{z}{ }_{X / H}\right)^{G}$ and $\beta$ for the inclusion of this into $\left(\pi_{*} \Omega^{z}{ }_{X}\right)^{G} \cdot \alpha_{y}$ is always an isomorphism. To see this note that from Theorem 1 of Chapter $I$ it suffices to prove $\alpha$ an isomorph ism at every point of codimension 1 passing through $y$. Now if $\left(\tau_{*} \Omega_{X / H}\right)^{G}$ and $\beta^{\prime}$ are defined by the pull-back diagram

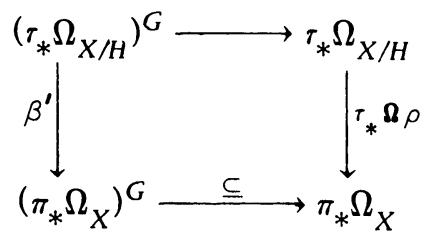

and $\alpha^{\prime}: \Omega_{X / G} \rightarrow\left(\tau_{*} \Omega_{X / H}\right)^{G}$ is the unique map such that $\beta^{\prime} \alpha^{\prime}=\Omega \pi=\tau_{*} \Omega \rho \Omega \tau$, then we have the commutative diagram

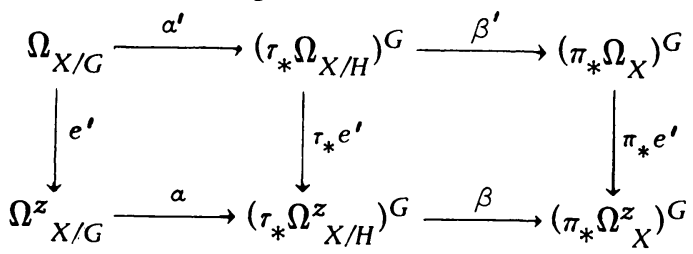

in which the vertical arrows are isomorphisms at each point of codimension 1 on $X / G$. Thus $a_{y}$ is an isomorphism iff $a^{\prime}$ is an isomorphism at each point of codimension 1 passing through $y$. As $H$ contains the ramification group of $x, \tau$ is étale at $z$ (see [2, SGA 1, Exp. V, p. 8]) and so $\Omega_{X / G, y} \otimes \tau_{*} \mathcal{O}_{X / H, y} \rightarrow^{\tau}{ }_{*} \Omega_{X / H, y}$ is an isomorphism. (The tensor product is over $\mathcal{O}_{X / G, y}$.) $G$ acts on both these modules in a fashion compatible with the isomorphism, so $\Omega_{X / G, y} \otimes\left(\tau_{*} \Theta_{X / H}\right)_{y}^{G}$ $\rightarrow\left(\tau_{*} \Omega_{X / H}\right)_{y}^{G}$ is an isomorphism. But $\left(\tau_{*} \mathcal{O}_{X / H}\right)^{G} \cong \mathcal{O}_{X / G}$, so $\left(\tau_{*} \Omega_{X / H}\right)^{G}{ }_{y} \cong$ $\Omega_{X / G, y}$ and this isomorphism is in fact $\alpha^{\prime}{ }_{y}$. From this it follows that $\alpha^{\prime}$ is an isomorphism at every point of codimension 1 passing through $y$, and so $\alpha_{y}$ is an isomorphism as claimed.

Proof of the theorem has now been reduced to showing that $\beta_{y}$ is an isomorphism iff $\Omega^{z} \rho_{z}$ is an isomorphism. One direction is clear: by Corollary 2 to Lemma 4 of Chapter I both $\Omega^{z}{ }_{X / H, z}$ and $\left(\rho_{*} \Omega^{z}{ }_{X}\right)^{H}{ }_{z}$ may be considered to be 
subsets of $\Omega^{z}(K)$ and $\Omega^{z} \rho_{z}$ is then an inclusion. Moreover intersecting each of these with $\Omega^{z}\left(K^{G}\right)=\Omega^{z}(K)^{G}$ gives $\left(\tau_{*} \Omega^{z}{ }_{X / H}\right)^{G}$ and $\left(\pi_{*} \Omega^{z}{ }_{X}\right)_{y}^{G}$ respectively, while $\Omega^{z} \rho_{z}$ restricted to this subset is $\beta_{y}$. So $\Omega^{z} \rho_{z}$ an isomorphism implies $\beta_{y}$ an isomorphism.

For the converse it suffices to prove that $\beta_{y}{ }^{\wedge}$ an isomorphism implies $\Omega^{z} \rho_{z}{ }^{\wedge}$ an isomorphism. Let $x_{1}, \cdots, x_{g}$ be the distinct points of $X$ lying over $y$. Let $z_{i}=\rho\left(x_{i}\right)$, so $z_{1}, \cdots, z_{g}$ are the distinct points of $X / H$ lying over $y$. Then from Lemma 1, $\left(\left(\tau_{*} \Omega_{X / H, y}^{z}\right)^{G}\right)^{\wedge}=\left(\left(\tau_{*} \Omega_{X / H, y}^{z}\right)^{\wedge}\right)^{G}$ and $\left(\left(\pi_{*} \Omega_{X, y}^{z}\right)^{G}\right)^{\wedge}=$ $\left(\left(\pi_{*} \Omega_{X, y}^{z}\right)^{\wedge}\right)^{G}$. Moreover

$$
\tau_{*} \Omega_{X / H, y}^{z} \cong \prod_{i=1}^{g} \Omega_{X / H, z_{i}}^{z}, \text { and } \pi_{*} \Omega_{X, y}^{z} \cong \prod_{i=1}^{g} \rho_{*} \Omega_{X, z_{i}}^{z}
$$

with $\tau_{*} \Omega^{z} \rho_{y}{ }^{\wedge}$ the product of the maps $\Omega^{z} \rho_{z_{i}}{ }^{\wedge}$. But $\beta_{y}{ }^{\wedge}$ is the restriction of $\tau_{*} \Omega^{z} \rho_{y}{ }^{\wedge}$ to $\left(\left(\tau_{*} \Omega^{z}{ }_{X / H, y}\right)^{\wedge}\right)^{G}$.

$G$ acts transitively on $z_{1}, \cdots, z_{g}$ so we may choose $\sigma_{1}, \cdots, \sigma_{g}$ in $G-\sigma_{1}$ being the identity-so that $\sigma_{i}\left(z_{1}\right)=z_{i}$. These are a complete set of representatives for a left coset decomposition of $G$ by $H$.

Let $\omega_{1} \in\left(\rho_{*} \Omega^{z}{ }_{X, z_{1}}{ }^{n}\right)^{H}$. If we can show that $\omega_{1}$ is in the image of $\Omega^{z} \rho_{z_{1}}$ the proof will be complete. Put $\omega_{i}=\sigma_{i}\left(\omega_{1}\right)$ and $\omega=\left(\omega_{1}, \cdots, \omega_{g}\right)$ so that $\omega \in\left(\pi_{*} \Omega^{z}{ }_{X, y}{ }^{\wedge}\right)^{G}$. When $\beta_{y}{ }^{\wedge}$ is an isomorphism there is $\omega^{*}=\left(\omega_{1}{ }^{*}, \cdots, \omega_{g}{ }^{*}\right)$ so that $\beta_{y}{ }^{\wedge}\left(\omega^{*}\right)=\omega$. But then $\Omega^{z} \rho_{z_{1}}{ }^{\wedge}\left(\omega_{1}{ }^{*}\right)=\omega_{1}$ as desired.

For the remainder of the paper the base field $k$ is algebraically closed.

Theorem 2. Let $X, G$ and $\pi$ be as in Theorem 1. For $x$ any point in $X$ write $H$ for its ramification group, $\rho: X \rightarrow X / H$ for the associated quotient map and $z=\rho(x)$. Then $\Omega^{z} \pi: \Omega_{X / G}^{z} \rightarrow\left(\pi_{*} \Omega^{z}{ }_{X}\right)^{G}$ is an isomorphism iff for each $x$ in $X, \Omega^{z} \rho_{z}: \Omega_{X / H, z}^{z} \rightarrow\left(\rho_{*} \Omega_{X, z}^{z}\right)^{H}$ is an isomorphism.

The "only if" part of this Theorem is contained in the proof of Theorem 1 where the corresponding statement needed only that the decomposition group contain the ramification group. For the other part we need the following:

Lemma 2. With the same notation as in Theorem 2 there is a closed point $x_{0}$ in the closure of $x$ which also bas $H$ as ramification group.

The proof is clear once we note that $G$ is finite and the ramification locus of any $\sigma \in G$ is closed.

Proof of Theorem 2. Let $x \in X, H$ and $x_{0}$ be as above. Since $k$ is algebraically closed, $H$ is the decomposition group of $x_{0}$, and so $\Omega^{z} \pi$ an isomorphism implies (by Theorem 1) that $\Omega^{z} \rho_{z_{0}}$ is an isomorphism. But $\Omega^{z} \rho_{z}$ is gotten by localizing $\Omega^{z} \rho_{z_{0}}$ at the prime ideal corresponding to $z$ in $\mathcal{O}_{X / H, z_{0}}$ and so is also an isomorphism. 
3. The tamely ramified case. By definition $\pi: X \rightarrow X / G$ is tamely ramified at $x$ iff the characteristic, $p$, of $k$ does not divide $b$, the order of the ramification group.

Theorem 3. If $\pi: X \rightarrow X / G$ is tamely ramified at every point of codimension 1 , then $\Omega^{z} \pi: \Omega_{X / G}^{z} \rightarrow\left(\pi_{*} \Omega^{z}{ }_{X}\right)^{G}$ is an isomorphism.

Proof. We need only show that for each point $x \in X$ of codimension 1 and with ramification group $H, \Omega^{z} \rho_{z}: \Omega^{z}{ }_{X / H, z} \rightarrow\left(\rho_{*} \Omega^{z}{ }_{X, z}\right)^{H}$ is an isomorphism. (As above $\rho: X \rightarrow X / H$ is the quotient map and $z=\rho(x)$.) Now

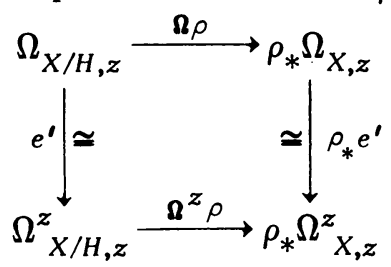

is commutative and the vertical maps are isomorphisms since $z$ is a point of codimension 1. As $\rho_{*} e^{\prime}$ is compatible with the actions of $H$ it will suffice to prove that $\Omega_{\rho:} \Omega_{X / H} \rightarrow\left(\rho_{*} \Omega_{X, z}\right)^{H}$ is surjective (since $\Omega^{z} \rho$ is always injective).

Note that both $\mathcal{O}_{X, x}$ and $\mathcal{O}_{X / H, z}$ are discrete valuation rings and so $\Omega_{X, x}$ and $\Omega_{X / H, z}$ are free modules of finite rank. Moreover since $H$ is the ramification group of $x, \rho_{*} \mathcal{O}_{X, z}=\mathcal{O}_{X, x}, \rho_{*} \Omega_{X, z}=\Omega_{X, x}$ and $\rho_{*} \Omega^{z}{ }_{X, z}=\Omega^{z}{ }_{X, x}$.

For convenience in the remainder of the proof we will abbreviate our notation to $\mathcal{O}_{X, x}=\mathcal{O}_{x}, \Omega_{X / H, z}^{z}=\Omega_{z}^{z}$, etc.

Now since $x$ is the only point of $X$ lying over $z, \mathcal{O}_{x}$ is the integral closure of $\mathcal{O}_{z}$ in $K$. Thus if $u$ is a generator of the maximal ideal of $\mathcal{O}_{\boldsymbol{x}}$, then $u^{b}$ is a generator of the maximal ideal of $\mathcal{O}_{z}$. As $p \nmid \not b$ and $k$ is algebraically closed $T^{b}-u^{b}$ is irreducible and is the minimal polynomial of $u$. This shows that the conjugates of $u$ over $k\left(u^{b}\right)$ are $u, \xi u, \cdots, \xi^{b-1} u$, where $\xi$ is a primitive $b$ th root of unity, and so. $H$ is a cyclic group with a generator $\sigma$ acting on $\mathcal{O}_{x}=$ $\mathcal{O}_{z}[u]$ as $\sigma(u)=\xi u$.

To show that $\Omega \rho$ is an epimorphism consider an arbitrary element $\omega$ in $\left(\rho_{*} \Omega_{X, z}\right)^{H} \cdot \omega=f_{1} d g_{1}+\cdots+f_{s} d g_{s}+f d u$ with $f, f_{i} \in \mathcal{O}_{x}$ and $g_{i} \in \mathcal{O}_{z} \cdot \omega$ is invariant under $H$, i.e., $\sigma(\omega)=\omega$, so $b \omega=\sum_{i=1}^{s} \sum_{j=0}^{b-1} \sigma^{j(}\left(f_{i}\right) d g_{i}+\sum_{j=0}^{b-1} \sigma^{j}(f) \xi^{j} d u$ and $\omega=\sum_{i=1}^{s} f_{i}^{*} d g_{i}+f^{*} d u$ where $f_{i}^{*}=b^{-1} \sum_{j=0}^{i=1} \sigma^{j}\left(f_{i}\right)$ and $f^{*}=$ $b^{-1} \sum_{j=0}^{b-1} \sigma^{j}(f) \xi^{j}$. Now, $f_{i}^{*} \in\left(\Theta_{x}\right)^{H}=\mathcal{O}_{z}$, so $\sum_{i=1}^{s} f_{i}^{*} d g_{i}$ is in the image of $\Omega \rho$ and $f^{*} d u$ is in $\left(\Omega_{x}\right)^{H}$. Thus we have only to show that if $f d u$ is in $\left(\Omega_{x}\right)^{H}$, then it is in the image of $\Omega \rho$. But $f$ may be written uniquely as $f=\sum_{i=0}^{b-1} a_{i} u^{i}$ with $a_{i} \in \mathcal{O}_{z^{\circ}}$. Now $0=\sigma(f d u)-f d u=\sum_{i=0}^{b-1} a_{i}\left(\xi^{i+1}-1\right) u^{i} d u$. Moreover $\Omega_{x}$ is a free $\mathcal{O}_{x}^{i}$-module and so a torsion-free $\mathcal{O}_{z}$-module, whence $\sum_{i=0}^{b-1} a_{i}\left(\xi^{i+1}-1\right) u^{i}=0$. But then $a_{i}\left(\xi^{i+1}-1\right)$ must be 0 for $i=0, \cdots, b-1$ and so $a_{i}=0$ for 
$i=0, \cdots, b-2$ while $a_{b-1}$ is arbitrary ( $\xi$ is a primitive $b$ th root of unity). Thus $f d u=a u^{b-1} d u$ with $a \in \mathcal{O}_{z}^{-1}$. But $a u^{b-1} d u=b^{-1} a d\left(u^{b}\right)$ which is in the image of $\Omega \rho$ as desired.

Corollary. Let $X, G, \pi$ be as above. Suppose both $X$ and $X / G$ are nonsingular and that $\pi$ is tamely ramified in codimension 1 . Then $\Omega_{\pi}: \Omega_{X / G} \rightarrow$ $\left(\pi_{*} \Omega_{X}\right)^{G}$ is an isomorphism.

Proof. If $X$ and $X / G$ are nonsingular, then $\Omega_{X}$ and $\Omega_{X / G}$ are locally free and so each $e^{\prime}$ is an isomorphism compatible with the actions of $G$. Apply the theorem.

Note. If $X$ and $X / G$ are nonsingular but $\pi$ is wildly ramified $\Omega \pi$ will not in general be an isomorphism. A bountiful supply of examples is to be found in Chapter III.

\section{Examples.}

Proposition 2. Let $X$ be a variety for which $t \Omega_{X}$, the torsion submodule of $\Omega_{X}$, is zero. Then the kernel of $\Omega_{\pi}$ is $t \Omega_{X / G}$.

Proof. Look at the commutative diagram

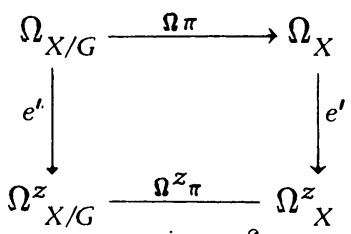

and remember that $\Omega^{z} \pi$ is a monomorphism $(\$ 1)$ and the kernel of $e^{\prime}$ is the torsion submodule. (This follows from Proposition 3 of Chapter I.)

All of the examples offered here are affine, so we will freely confuse varieties with their affine rings and (coherent) sheaves with their modules of global sections.

Example 1. $\Omega \pi: \Omega_{X / G} \rightarrow\left(\Omega_{X}\right)^{G}$ may be a monomorphism but not an epimorphism. Suppose the characteristic of $k$ is not 2 and let $\sigma$ be the automorphism of $A=k\left[T_{1}, T_{2}\right]$ given by $\sigma\left(T_{i}\right)=-T_{i}$. This gives an action of the cyclic group of order 2 on the affine plane, and $A^{G}$ is $k\left[T_{1}{ }^{2}, T_{1} T_{2}, T_{2}{ }^{2}\right] \cong$ $k[U, V, W] /\left(U V-W^{2}\right)$, i.e., $X / G$ is the cone. This is a normal hypersurface so according to Lipman [4, Proposition 8.1] $t \Omega_{X / G}=0$, and thus $\Omega \pi$ is a monomorphism. It is not an epimorphism: $X d Y$ is in $\left(\Omega_{X}\right)^{G}$ but is clearly not in the image of $\Omega \pi$.

Note that here $\pi$ is ramified only at the origin, so it is unramified in codimension 1 and thus $\Omega^{z}: \Omega^{z}{ }_{X / G} \rightarrow\left(\Omega^{z}{ }_{X}\right)^{G}$ is an isomorphism. This shows that both $e: \Omega_{X / G} \rightarrow \Omega_{X / G}{ }^{* *}$ and $e^{\prime}: \Omega_{X / G} \rightarrow \Omega^{z}{ }_{X / G}$ are monomorph isms but not epimorphisms. 
Example 2. $\Omega \pi$ may be neither a monomorphism nor an epimorphism. This example is taken from Suzuki [10]. Suppose the characteristic of $k$ is not 3 and let $\sigma$ be the automorphism of $A=k\left[T_{1}, T_{2}\right]$ defined by $\sigma\left(T_{i}\right)=\xi T_{i}$ where $\xi$ is a primitive cube root of unity. This gives an action of the cyclic group of order 3 on the affine plane and $A^{G}$ is $k\left[T_{1}{ }^{3}, T_{1}{ }^{2} T_{2}, T_{1} T_{2}{ }^{2}, T_{2}{ }^{3}\right] \cong$ $k[T, U, V, W] /\left(U^{2}-T V, V^{2}-U W, U V-T W\right)$, i.e., $X / G$ is the cone over the twisted cubic.

To show that $\Omega \pi$ is not a monomorphism it suffices to show that $\Omega_{X / G}$ has torsion. Consider the nonzero differential $\omega=-W d T+2 V d U-U d V$ in $\Omega_{X / G}$. Then $T \omega=-T W d T+2 T V d U-T U d V=-U V d T+2 U^{2} d U-T U d V=U d\left(U^{2}-T V\right)=0$.

To see that $\Omega \pi$ is not an epimorphism note that $T_{1}{ }^{2} d T_{1}$ is invariant under $\sigma$, but clearly not in the image of $\Omega \pi$.

Again $\pi$ is ramified only at the origin, so $\Omega^{z} \pi$ is an isomorphism as is also $e^{\prime}{ }_{X}: \Omega_{X} \rightarrow \Omega^{z}{ }_{X}$ (because $X$ is nonsingular). Thus $e_{X / G}^{\prime}$ and $e_{X / G}$ are both neither monomorphisms nor epimorphisms.

I do not know whether $\Omega \pi$ can be an epimorphism without being a monomorphism.

Our final result in this chapter relates the failure of $\Omega \pi: \Omega_{X / G} \rightarrow\left(\Omega_{X}\right)^{G}$ to be an isomorphism to the different.

Defintion. Let $f: X \rightarrow Y$ be a map of varieties. The different of $Y$ over $X$ is the annihilator of the relative module of differentials $\Omega_{Y} / X^{*}$. We denote it by Dी $_{Y \cdot / X}$. For more information see [2, SGA 1, Exp. 1].

Let $X, G$ and $\pi$ be as usual. Let $x \in X$ be a point of codimension $1, y=$ $\pi(x)$ and let $\mathcal{D}$ be the different of $X$ over $X / G$ at $x . \mathcal{O}_{x}$ is a discrete valuation ring and $\mathscr{D}=\mathrm{m}_{x}{ }^{n}$ for some nonnegative integer $n, \mathrm{~m}_{x}$ being the maximal ideal of $\mathcal{O}_{x}$. Suppose $\omega^{\prime} \in \Omega_{K_{K}}\left(=\Omega_{X / G, y_{0}}\right.$ where $y_{0}$ is the generic point of $\left.X / G\right)$ and that $\Omega \pi\left(\omega^{\prime}\right)=\omega \in \Omega_{x}$. Then we have

Proposition 3. ord $_{y}\left(\omega^{\prime}\right) \geq-n$.

Proof. We may consider $\Omega_{x} \subseteq \Omega_{K}$ and $\Omega_{y} \subseteq \Omega_{K} \subseteq \subseteq \Omega_{K}$ and can then restate the proposition as saying that $m_{y}{ }^{n}\left(\Omega_{x} \cap \Omega_{K} G\right) \subseteq \Omega_{y}$. This is the same as saying that $\mathrm{m}_{y}{ }^{n}\left(\Omega_{x} \cap \Omega_{K} G\right)$ is contained in the image of the map $\Omega_{y} \otimes \Theta_{y} \rightarrow \Omega_{y} \otimes K$ $\cong \Omega_{K^{\prime}}$. (The tensor products here and throughout this proof are over $\Theta_{y}$.) But $\Omega_{y} \otimes \Theta_{y}=\left(\Omega_{y} \otimes \Theta_{x}\right) \cap\left(\Omega_{y} \otimes K^{G}\right)$, so it suffices to show that $m_{y}{ }^{n}\left(\Omega_{x} \cap \Omega_{K^{G}}\right)$ is in the image of $\Omega_{y} \otimes K^{G} \rightarrow \Omega_{y} \otimes K \cong \Omega_{K}$ and in the image of $\Omega_{y} \otimes \Theta_{x} \rightarrow$ $\Omega_{y} \otimes K \cong \Omega_{K}$. Since $\Omega_{y} \otimes K^{G}=\Omega_{K} G$, the image of the first map is $\Omega_{K}{ }^{\prime}$, while for the second map we have the sequence $\Omega_{y} \otimes \Theta_{x} \rightarrow \Omega_{x} \rightarrow \Omega^{\prime}{ }_{x} \stackrel{K^{G}}{\rightarrow} 0$ (where we have written $\Omega^{\prime}$ for the relative module of differentials of ${ }^{x} X$ over $X / G)$ and, as $\supseteqq$ annihilates $\Omega_{x}^{\prime}$, we have $m_{x}{ }^{n} \Omega_{x}$ in the image of $\Omega_{y} \otimes \Theta_{x} \rightarrow \Omega_{x}$. 
Now $m_{y}^{n} \Omega_{x} \subseteq m_{x}{ }^{n} \Omega_{x}$ and

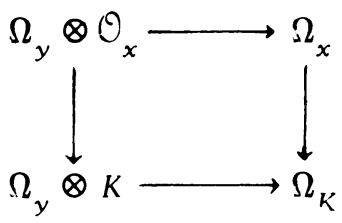

commutes, so we have the desired relation.

Example 3. (Poles of maximum possible order occur.) Suppose the characteristic of $k$ is a prime $p$ and let $A=k\left[T_{1}, T_{2}\right]$. Take $G$ to be a cyclic group of order $p$ generated by the automorphism $\sigma$ which acts on $A$ via $\sigma\left(T_{1}\right)=T_{1}$ and $\sigma\left(T_{2}\right)=T_{2}+T_{1}{ }^{p}$. Then $A^{G}=k\left[T_{1}, U\right]$ where $U=T_{2}{ }^{p}-T_{1}{ }^{p(p-1)} T_{2}$ (this is proved in Chapter III). Let $X$ be $\operatorname{Spec} A$ so $X / G=\operatorname{Spec} A^{G}$. Take $x$ to be the point $\left(T_{1}\right)$ of codimension 1 in $X$ and let $y=\pi(x)$. Consider the dif-

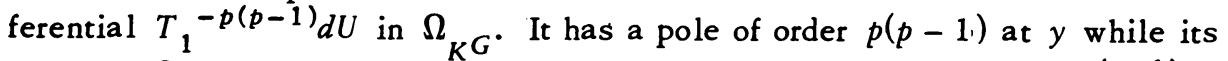
image in $\Omega_{K}$ is $d T_{2}$ which is regular at $x$. But in this case $\mathscr{D}=\left(T_{1} p(p-1)\right)$, so the above differential has a pole of maximum possible order.

In general the bound given by this Proposition will not be obtained. This is partly because 9 measures only ramification. For example if $\pi$ is ramified but tamely ramified in codimension 1 , then $\mathfrak{D}_{Y, / X}$ will be nonzero while Theorem 3 assures us that any differential in $\Omega_{{ }_{K} G}$ whose image is in $\Omega_{x}, x$ of codimension 1 , will be in $\Omega_{y}(y=\pi(x))$. Even in the case where $\pi$ is totally and wildly ramified at $x$ the bound provided by Proposition 2 may not be obtained. This will appear from the computations of Chapter III.

\section{CHAPTER III. THE WILDLY RAMIFIED CASE}

In Chapter II we showed that if the finite group $G$ acts on a nonsingular variety $X$ in such a fashion that the quotient map $\pi: X \rightarrow X / G$ is tamely ramified in codimension 1 , then $\Omega^{z} \pi: \Omega^{z}{ }_{X / G} \rightarrow\left(\pi_{*} \Omega^{z}{ }_{X}\right)^{G}$ is an isomorphism. By contrast if $\pi$ is wildly ramified in codimension 1 the situation is very complicated. For the case where the ramification group at each closed point acts "linearly" (definition below) our principal result, Theorem 1, gives necessary and sufficient conditions that $\Omega^{z} \pi$ be an isomorphism.

Throughout this chapter $k$ is algebraically closed.

Let $\mathcal{O}$ be a regular local ring so that its completion $\mathcal{O}^{\wedge}$ is a formal power series ring.

Definition. A group of automorphisms of $\Theta$ acts linearly iff the induced action on $\mathcal{O}^{\wedge}$ is linear (in the obvious sense).

We will also need the

Definition. Let $H$ be a group of automorphisms of a local ring $\mathcal{O}$. Let $Q$ be the class of ideals $q$ of $\mathcal{O}$ with the property that $H$ is the decomposition 
group of every minimal prime ideal contained in $q$. Then $Q$ has a maximal element which we call the decomposition ideal of $H$.

The primary purpose of this chapter is to prove

Theorem 1. Let $X$ be a nonsingular k-variety of dimension $n$, and let $G$ be a finite group of $k$-automorphisms of $X$ with the ramification group of eac $b$ closed point acting linearly on the local ring of that point. If $x_{0}$ is a closed point of $X$ and $y_{0}=\pi\left(x_{0}\right)$, then $\Omega^{z} \pi_{y_{0}}: \Omega_{X / G, y_{0}}^{z} \rightarrow\left(\pi_{*} \Omega^{z}{ }_{X}\right)_{y_{0}}$ is an isomorphism iff for each point $x$ of codimension'1 passing through $x_{0}$ either:

(a) the ramification group $H$ of $x$ bas order prime to $p$, the characteristic of $k$; or

(b) there is a positive integer $r$ so that the $p$ subgroup $H^{\prime}$ of $H$ is the direct product of $t$ ( $=$ the dimension or corank of the decomposition ideal of $H$ ) subgroups of order $p^{r}$, the index of $H^{\prime}$ in $H$ is $p^{r}-1$, and if $\tau \in H$ generates $H / H^{\prime}$ (which is always cyclic-see the paragraph preceding Proposition 1 below), then each of the direct factors $H_{i}$ of $H^{\prime}$ is invariant under conjugation by $\pi$.

Corollary. With $X$ and $G$ as in Theorem $1, \Omega^{z} \pi: \Omega^{z}{ }_{X / G} \rightarrow\left(\pi_{*} \Omega^{z}{ }_{X}\right)^{G}$ is an isomorphism iff the ramification group of every point of codimension 1 bas the form specified in (a) or (b) of Theorem 1.

The proof of Theorem 1 consists of a series of reductions followed by a computation in affine space. We begin with $X$ and $G$ as in Theorem 1 and let $x_{0}$ be a closed point in $X, y_{0}=\pi\left(x_{0}\right), G_{0}$ the ramification group of $x_{0}, \rho: X \rightarrow$ $X / G_{0}$ the quotient map and $z_{0}=\rho\left(x_{0}\right)$. By Theorem 2 of Chapter II, $\Omega^{z} \pi_{y_{0}}$ : $\Omega_{X / G, y_{0}}^{z} \rightarrow\left(\pi_{*} \Omega_{X}^{z}\right)_{y_{0}}^{G}$ is an isomorphism iff $\Omega^{z} \rho_{z_{0}}: \Omega_{X / G_{0}, z_{0}}^{z} \rightarrow\left(\rho_{*} \Omega_{X}^{z}\right)^{G_{0}}{ }_{z_{0}}$ is an is omorphism.

To reduce the problem to considering affine space let $\mathcal{O}$ be the local ring of $x_{0}$ and $\mathcal{O}^{\wedge}$ its completion. By assumption there is a basis $b_{1}, \cdots, b_{n}$ for the maximal ideal $\mathrm{m}^{\wedge}$ of $\mathcal{O}^{\wedge}$ so that each $\sigma$ in $G_{0}$ acts on the $b_{j}$ via $\sigma^{n}\left(b_{j}\right)=$ $\Sigma \sigma_{i j} b_{i}$ with $\left(\sigma_{i j}\right)$ a nonsingular matrix with coefficients in $k$. Define an action of $G_{0}$ on the $n$-dimensional affine space $\mathbf{A}^{n}=\operatorname{Spec} k\left[X_{1}, \cdots, X_{n}\right]$ by $\sigma\left(X_{j}\right)=$ $\Sigma \sigma_{i j} X_{i}$ for each $\sigma$ in $G_{0}$. Let $\alpha: \mathbf{A}^{n} \rightarrow \mathbf{A}^{n} / G_{0}$ be the quotient map, and write o for the "origin" of $A^{n}$, i.e. the point $\left(X_{1}, \cdots, X_{n}\right)$. Write $\mathcal{O}_{0}$ for the local ring of $\mathrm{o}$ on $\mathrm{A}^{n}$. Then we have a $G_{0}$-compatible morphism $\mathcal{O}_{0} \rightarrow \mathcal{O}$ which induces a $G_{0}$-compatible isomorphism $\mathcal{O}_{0}^{\wedge} \rightarrow \mathcal{O}^{\wedge}$ on the completions. Thus we have also $\Omega^{z}{ }_{\mathrm{A}^{n, 0}} \cong \Omega_{X, x_{0}}{ }^{\wedge}$. Because this too is $G_{0}$-compatible, Lemma 1 of Chapter II tells us that $\Omega^{z} \rho_{z_{0}}{ }^{\wedge}$ is an isomorphism iff $\Omega^{z} a_{\mathrm{p}}{ }^{\wedge}: \Omega^{z}{ }_{\Lambda^{n} / G_{0, p}}{ }^{\wedge} \rightarrow$ $\left(a_{*} \Omega_{\Lambda^{n}}^{z}\right)^{G_{0}} \wedge(p=\alpha(0))$ is an isomorphism. But $\Omega^{z} \rho_{z_{0}}^{p}$ and $\Omega^{z} \alpha_{p}$ are isomorphisms iff the ir completions are isomorphisms, so $\Omega^{z} \rho_{z_{0}}$ is an isomorphism 
iff $\Omega^{z} \alpha_{p}$ is an isomorphism. Now $\Omega^{z} \alpha_{p}$ is an isomorphism exactly when $\Omega^{z} \alpha$ is an isomorphism at every point of codimension 1 passing through p. And of course we need only check those points of codimension 1 where $\alpha$ is ramified. But as $G_{0}$ acts linearly on $A^{n}$ the only points of codimension 1 where $\alpha$ is ramified are the generic points of a finite number of hyperplanes through o. Thus $\Omega^{z} \alpha$ is an isomorphism iff it is an isomorphism at every point which is the image of the generic point of one of the hyperplanes along which $\alpha$ is ramified. Let $\mathbf{h}$ be the generic point of such a hyperplane, $H$ the ramification group of $\mathrm{h}, \beta: \mathbf{A}^{n} \rightarrow \mathbf{A}^{n} / H$ the quotient map and $q=\beta(\mathrm{h})$. Then from Chapter II we know that $\Omega^{z} \alpha$ is an isomorphism at $\alpha(h)$ iff $\Omega^{z} \beta_{q}$ is an isomorphism. Thus we are finally reduced to showing that $\Omega^{z} \beta_{\mathbf{q}}$ is an isomorphism iff the conditions of Theorem 1 hold. Note that as $\mathcal{O}$ and $\mathcal{O}_{0}$ have isomorphic completions, the $\varepsilon^{\text {roup }}$ $H$ above is also the ramification group of some point of codimension 1 passing through the closed point $x_{0}$, and conversely every subgroup of $G_{0}$ which is the ramification group of a point of codimension 1 passing through $x_{0}$ is also the ramification group of a hyperplane passing through $o$. So now we can say: Theorem 1 will be proved if we show that whenever $H$ is a finite group of linear automorphisms of $\mathbf{A}^{n}$ ramified at one hyperplane and $\beta$ and $q$ are as above, then $\Omega^{z} \beta_{\mathbf{q}}$ is an isomorphism iff $H$ satisfies one of the two conditions in Theorem 1 .

Our problem is now an exercise in commutative algebra. We have a finite group of linear automorphisms of the ring $A=k\left[X_{1}, \cdots, X_{n}\right]$ which is ramified at one minimal prime ideal. As the group $H$ acts linearly we may-by a suitable change of coordinates-assume that the minimal prime is $\left(X_{n}\right)$. We put $q=$ $\left(X_{n}\right) \cap A^{H}$ and note that $H$ is the ramification group of the discrete valuation ring $A_{\left(X_{n}\right)}$ over $A^{H}{ }_{q}$. From the classical ramification theory (Zariski and Samuel [12, Chapter $5, \S 10$, Theorem 25]), $H$ has a normal subgroup $H^{\prime}$ whose order is a power of $p$ and whose quotient group $H / H^{\prime}$ is cyclic of order $m$ prime to $p$. We note in particular that there is an element $\tau$ of order $m$ in $H$ whose coset generates $H / H^{\prime}$. By another change of coordinates we may assume that $\tau$ acts as $\tau\left(X_{n}\right)=\xi X_{n}$ ( $\xi$ being a primitive $m$ th root of unity) and $\tau\left(X_{i}\right)=X_{i}$ for $i<n$. Indeed simply note that as $\tau$ is ramified at $\left(X_{n}\right), r\left(X_{n}\right)-X_{n}=$ $(\xi-1) X_{n}$ for some $\xi$ in $k$, so $\tau\left(X_{n}\right)=\xi X_{n}$ and as $\tau$ is of order $m$, $\xi$ is a primitive $m$ th root of unity. For $i<n$ note that if $Y_{i}=(1 / m) \Sigma \tau^{j}\left(X_{i}\right)$ (the sum being from $j=0$ to $j=m-1$ ), then $Y_{i}$ is a linear combination of the $X_{j}$ 's (and conversely), and $\tau\left(Y_{i}\right)=Y_{i}$. Henceforth we assume that $\tau\left(X_{n}\right)=\xi X_{n}$ and $\tau\left(X_{i}\right)$ $=X_{i}$ for $i<n$.

Proposition 1. With the notation above, $H^{\prime}$ is a direct product of cyclic groups of order $p$. 
Proof. It suffices to show that if $\sigma$ is in $H^{\prime}$, then $\sigma$ has the form $\sigma\left(X_{i}\right)=$ $X_{i}+a_{i} X_{n}, a_{i} \in k$, for $i<n$ and $\sigma\left(X_{n}\right)=X_{n}$, as such a $\sigma$ is of order $p$ and any two such commute. Now any $\sigma$ in $H^{\prime}$ is ramified at $\left(X_{n}\right)$, so $\sigma\left(X_{n}\right)=a X_{n}$ for some $a$ in $k$. But the order of $\sigma$ is a power of $p$, so $a^{p^{r}}=1$ for some $r$, whence $a=1$. For $i<n, \sigma\left(X_{i}\right)-X_{i} \in\left(X_{n}\right)$, i.e. $\sigma\left(X_{i}\right)=X_{i}+a_{i} X_{n}$ as desired.

Note that any $\sigma$ in $H^{\prime}$ is completely determined by its associated vector $\mathbf{a}=\left(a_{1}, \cdots, a_{n-1}\right)$ in $k^{n-1}$.

Definition. Two elements $\sigma$ and $\sigma^{\prime}$ are of the same type iff their associated vectors $\mathbf{a}$ and $\mathbf{a}^{\prime}$ in $k^{n-1}$ are linearly dependent.

Lemma $1 . H^{\prime}$ can be decomposed as a direct sum of subgroups $H_{1}, \cdots, H_{t}$ with each $H_{i}$ stable under conjugation by $\tau$ and consisting entirely of elements of a single type.

Proof. Let $\sigma$ be any nonidentity element of $H^{\prime}$. Put $H_{1}$ equal to the subgroup of all elements of the same type as $\sigma$. This is stable under conjugation by $\tau$, as the effect of conjugation on the associated vector is multiplication by $\xi$. Now suppose we have gotten subgroups $H_{1}, \cdots, H_{i}$ stable under conjugation by $\tau$, each containing only elements of the same type, and with $H_{1} \oplus \ldots$ $\oplus H_{i}$ a subgroup of $H^{\prime}$. If this is not already $H^{\prime}$, then there is an element $\sigma$ which is not in this direct sum. Let $H_{i+1}$ be the subgroup of $H^{\prime}$ generated by the orbit of $\sigma$ under the conjugation of $\tau$ on $H^{\prime}$. This has only elements of the same type as $\sigma$, is stable under conjugation by $\tau$, and its intersection with $H_{1} \oplus \ldots \oplus H_{i}$ consists of just the identity. Thus $H_{1} \oplus \cdots \oplus H_{i+1}$ is also a subgroup of $H^{\prime}$. As $H^{\prime}$ is finite this process eventually exhausts $H^{\prime}$.

Definition. A subset of $H^{\prime}$ is independent iff the set of associated vectors in $k^{n-1}$ is linearly independent.

Note that in any decomposition of $H^{\prime}$, as in Lemma 1 , the number $t$ of summands of $H^{\prime}$ is always at least as large as the size of a maximal independent subset of $H^{\prime}$.

Lemma 2. In any decomposition of $H^{\prime}$ as in Lemma 1, if the order of $H_{i}$ is $p^{{ }^{i} i}$, then $m$ divides $p^{r_{i}}-1$.

Proof. The subgroup of $H$ generated by $\tau$ acts on $H_{i}$ by conjugation, and the orbit of every element other than the identity contains $m$ elements.

The next lemma will allow us to assume that $H^{\prime}$ has a maximal independent set of the largest possible size.

Lemma 3. If the size, $s$, of a maximal independent subset of $H^{\prime}$ is less than $n-1$, then there is a linear change of coordinates $\left(X_{1}, \cdots, X_{n}\right) \rightarrow$ $\left(Y_{1}, \cdots, Y_{n-1}, X_{n}\right)$ so that $H^{\prime}$ acts trivially on $Y_{s+1}, \cdots, Y_{n-1}$. 
Proof. By finite induction. Suppose the lemma has been proved for the subgroup $H^{\prime \prime}$ of all automorphisms dependent on the independent subset $\left\{\sigma_{1}, \cdots, \sigma_{q}\right\}$ via the new coordinates $\left(Z_{1}, \cdots, Z_{n-1}, X_{n}\right)$ and suppose $\left\{\sigma_{1}, \cdots, \sigma_{q+1}\right\}$ is also independent. Let $\mathrm{c}$ be the vector associated to $\sigma_{q+1}$ relative to the " $Z$ " coordinates. If $\mathbf{c}=\mathbf{o}$, we take $Y_{i}=Z_{i}$. If $\mathbf{c} \neq \mathbf{o}$, we may, by suitably reordering the coordinates, assume that $c_{q+1} \neq 0$. Then we define $Y_{i}=Z_{i}$ for $1 \leq i \leq q+1$ and $Y_{i}=c_{i} Z_{q+1}-c_{q+1} Z_{i}$ for $i>q+1$. As $c_{q+1} \neq 0$ this is a change of coordinates; moveover, for $i>q+1, \sigma_{q+1}\left(Y_{i}\right)=Y_{i}$. Now if $\sigma$ is dependent on $\left\{\sigma_{1}, \cdots, \sigma_{q+1}\right\}$, then $\sigma=\sigma^{\prime} \sigma^{\prime \prime}$ where $\sigma^{\prime \prime}$ is dependent on $\left\{\sigma_{1}, \cdots, \sigma_{q}\right\}$, while $\sigma^{\prime}$ is of the same type as $\sigma_{q+1}$. For $i>q+1, Y_{i}$ is invariant under any element of $H^{\prime \prime}$ (e.g. $\sigma^{\prime \prime}$ ) and also is invariant under $\sigma_{q+1}$, so it is invariant under $\sigma$.

Returning to our basic situation of $H$ acting on $k\left[X_{1}, \cdots, X_{n}\right]$, we know that the maximal possible size of an independent subset of $H^{\prime}$ is in $n-1$; if the actual size $s$ of a maximal independent subset is less than $n-1$, then Lemma 3 tells us that $H$ can be taken as acting trivially on $X_{s+1}, \cdots, X_{n}$. So we have an action of $H$ on $k\left[X_{1}, \cdots, X_{s}, X_{n}\right]$ and $\Omega^{z} \beta$ will be a $n$ isomorphism iff the map of Zariski differentials arising from the quotient map $\mathbf{A}^{s+1} \rightarrow \mathbf{A}^{s+1} / H$ is an isomorphism. Thus we may, and from now on do, assume that $H^{\prime}$ has an independent subset of size $n-1$.

Let $\left\{\sigma_{1}, \cdots, \sigma_{n-1}\right\}$ be such an independent subset of $H^{\prime}$. Put $K$ equal to the subgroup of $H$ generated by these $\sigma$ 's and by $\tau$. Then the $p$ subgroup $K^{\prime}$ of $K$ is the direct sum of the subgroups $K_{1}, \cdots, K_{n-1}$ where $K_{i}$ is the smallest subgroup of $K$ containing $\sigma_{i}$ and closed under conjugation by $\tau$. Moreover, each of the $K_{i}$ has order $p^{r}$ where $r$ is the smallest positive integer such that $m$ divides $p^{r}-1$.

The proposition from which we will derive Theorem 1 will come from comparing the quotients $\mathrm{A}^{n} / H$ and $\mathbf{A}^{n} / K$. The quotient map from $\mathbf{A}^{n}$ to $\mathbf{A}^{n} / H$ we've called $\beta$; for lack of a better name, the quotient map from $\mathrm{A}^{n}$ to $\mathrm{A}^{n} / K$ will be called $\mu$.

Proposition 2. Under the assumption that $H^{\prime}$ bas a maximal independent subset of size $n-1, \Omega^{z} \beta: \Omega_{\mathbf{A}^{n / H}}^{z} \rightarrow\left(\beta_{*} \Omega^{z}{ }_{\mathbf{A}^{n}}\right)^{H}$ is an isomorphism iff $H=K$ and $m=p^{r}-1$.

More precisely, if $m=p^{r}-1$, then $\Omega^{z} \mu: \Omega^{z}{ }_{A^{n / K}} \rightarrow\left(\mu_{*} \Omega^{z}{ }_{\Lambda^{n}}\right)^{K}$ is an isomorphism, while if $m<p^{r}-1$ then $\Omega^{z} \mu$ (and hence $\Omega^{z} \beta$ ) is not an epimorphism. Also if $K \neq H$, then $\Omega^{z} \beta$ is never an epimorphism.

Since all the varieties involved in Proposition 2 are affine and all the sheaves are coherent we may, and do, consider this as a result in commutative algebra. And this is how we will prove it. In particular we henceforth will speak of the 
ring $A=k\left[X_{1}, \cdots, X_{n}\right]$, the ring of invariants $A^{H}$, the inclusion $\beta: A^{H} \rightarrow A$, the $A$-module $\Omega_{A}^{z}$, etc., instead of the variety $\mathbf{A}^{n}$, the quotient variety $\mathbf{A}^{n} / H$, the quotient map $\beta$, the sheaf $\Omega^{z}{ }_{\mathbf{A}}$, etc.

The proof of Proposition 2 will consist of explicitly computing the ring $A^{K}$ and then the modules $\Omega^{z}{ }_{A}{ },\left(\Omega^{z}{ }_{A}\right)^{K}, \Omega^{z}{ }_{A} H$ and $\left(\Omega^{z}{ }_{A}\right)^{H}$. This will be accomplished with the aid of the following sequence of five lemmas.

Lemma 4. The coordinates in the polynomial ring $A$ can be chosen so that $K$ acts as $\tau\left(X_{i}\right)=X_{i}$ for $i<n$ and $\tau\left(X_{n}\right)=\xi X_{n}$, while $\sigma_{i}\left(X_{j}\right)=X_{j}$ for $i \neq j$, and $\sigma_{i}\left(X_{i}\right)=X_{i}+a_{i} X_{n}$ with $a_{i} \neq 0$.

Proof. Suppose we have gotten coordinates $Y_{1}, \cdots, Y_{k}, X_{k+1}, \cdots, X_{n}$ so that the $\sigma$ 's and $\tau$ act as desired as long as $i>k$ or $j>k$. Consider the independent set $\left\{\sigma_{1}, \cdots, \sigma_{k-1}\right\}$. The proof of Lemma 3 shows that we can change coordinates to $Y_{1}, \cdots, Y_{k-1}, X_{k}, X_{k+1}, \cdots, X_{n}$ with $X_{k}$ a linear combination of $Y_{1}, \cdots, Y_{k}$ in such a way that $\sigma_{i}\left(X_{k}\right)=X_{k}$ for $i=1, \cdots, k-1$ and $\sigma_{k}\left(X_{k}\right)$ $=X_{k}+a_{k} X_{n}$. Note that necessarily $a_{k} \neq 0$ for otherwise $\left\{\sigma_{1}, \cdots, \sigma_{n-1}\right\}$ would be dependent. Now $\sigma_{k}\left(Y_{j}\right)=Y_{j}+b_{j} X_{n}$ for some $b_{j}$. Put $Y_{j}^{\prime}=b_{j} X_{k}-a_{k} Y_{j}$. Then $\sigma_{k}\left(Y_{j}{ }^{\prime}\right)=Y_{j}{ }^{\prime}$. Thus we now have the coordinates $Y_{1}{ }^{\prime}, \cdots, Y_{k-1}{ }^{\prime}, X_{k}$, $\cdots, X_{n}$, for which the $\sigma$ 's and $\tau$ act as desired as long as $i>k-1$ or $j>k-1$. Since this process can be started with $k=n$ and must stop when $k=1$, we are done.

From now on we assume we have chosen such a coordinate system.

Lemma 5. If the automorphism $\sigma$ of the polynomial ring $k\left[X_{1}, \cdots, X_{n}\right]$ is given by $\sigma\left(X_{1}\right)=X_{1}+a X_{n}^{q}$ ( $a \in k, a \neq 0, q$ a positive integer) and $\langle\sigma\rangle$ is the group generated by $\sigma$, then $A^{(\sigma)}=k\left[U, X_{2}, \cdots, X_{n}\right]$ where $U=X_{1}{ }^{p}-$ $a^{p-1} X_{n}^{q(p-1)} X_{1}$.

Proof. Write $B$ for $k\left[U, X_{2}, \cdots, X_{n}\right]$, and note that $B \subset A^{(\sigma)}$. Moreover, $X_{1}$ is a root of the monic polynomial $P(T)=T^{p}-a^{p-1} X_{n}{ }^{q(p-1)} T-U$ over $B$, so $A$ is integral over $B$. Since $P(T)$ has degree $p$ and $\sigma$ has order $p$, the quotient field of $B$ is the field of invariants of the action of $\sigma$ on the quotient field of $A$. As $A$ is integrally closed, $B=A^{\langle\sigma\rangle}$ follows.

As every element of $K_{i}$ has the same type as $\sigma_{i}$, it follows that each $\sigma$ in $K_{i}$ acts on $A$ in the fashion $\sigma\left(X_{j}\right)=X_{j}$ if $i \neq j$, and $\sigma\left(X_{i}\right)=X_{i}+a_{\sigma} X_{n}$. Proposition 1 shows that $K_{i}$ is a direct sum of cyclic groups of order $p$.

The next three lemmas provide an inductive computation of $A^{K_{1}}$. To simplify the notation we use $\sigma_{1}, \cdots, \sigma_{r}$ to stand for independent generators of $K_{1}$ so that $K_{1}=\left\langle\sigma_{1}\right\rangle \oplus \cdots \oplus\left\langle\sigma_{r}\right\rangle$. Note that this use of the symbol $\sigma_{i}$ bas nothing in common with its earlier use. We write $K_{1 i}$ for the subgroup of $K_{1}$ generated by $\sigma_{1}, \cdots, \sigma_{i}$. 
Lemma 6. Suppose we know that, for $1 \leq i \leq s, A^{K_{1 i}}=k\left[U_{i}, X_{2}, \cdots, X_{n}\right]$

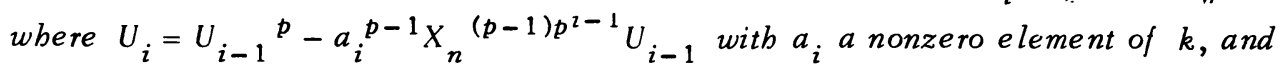
$U_{0}=X_{1}$. Suppose also that $\sigma$ is an automorphism of $A$ baving the form $\sigma\left(X_{j}\right)=$ $X_{j}$ for $j>1$ and $\sigma\left(X_{1}\right)=X_{1}+a X_{n}, a \neq 0$; that $\sigma\left(U_{s-1}\right)=U_{s-1}+c X_{n} p^{s-1}, c \neq 0$; and that $\sigma$ acts nontrivially on $A^{K_{1}}$. Then $\sigma\left(U_{s}\right)=U_{i s}+b X_{n} p^{s}$ with $b \neq 0$. In particular, if we assume that, for all $j>s, \sigma_{j}\left(U_{s-1}\right)=U_{s-1}+c_{j} X_{n}{ }^{p s-1}$ with $c_{j} \neq 0$, and that $\sigma_{j}$ acts nontrivially on $A^{K_{1 i}}$, then $\sigma_{j}\left(U_{s}\right)=U_{s}+b_{j} X_{n}{ }^{s}$ where $b_{j}=\left(c_{j}^{p}-c_{j} a_{s}^{p-1}\right)$. Moreover, it follows that $A^{K_{1, s+1}}=\left(A^{K_{1 s}}\right)^{\left(\sigma_{s+1}{ }^{\prime}\right.}=$ $k\left[U_{s+1}, X_{2}, \cdots, X_{n}\right]$ with $U_{s+1}=U_{s}-b_{s+1}^{p-1} X_{n}{ }^{(p-1) p^{s}} U_{s}$.

Proof. Since $\sigma\left(U_{s}\right)=U_{s}+b X_{n} p^{s}$ with $b=\left(c^{p}-c a_{s}^{p-1}\right)$-by computationand $\sigma\left(X_{j}\right)=X_{j}$ for $j>1, \sigma$ will act nontrivially on $A^{K_{1} s}$ iff $b \neq 0$. The remainder of the lemma follows from Lemma 5.

Once we prove that $\sigma_{s+1}$ acts nontrivially on $A^{K_{1 s}}$ (and this is done in Lemma 8) Lemma 6 provides an inductive computation of $A^{K_{1}}$.

Lemma 7. Suppose that $\sigma$ and $\sigma^{\prime}$ are two automorphisms of $A$ which act as $\sigma\left(X_{j}\right)=X_{j}=\sigma^{\prime}\left(X_{j}\right)$ for $j>1, \sigma\left(X_{1}\right)=X_{1}+a X_{n}{ }^{q q}$ and $\sigma^{\prime}\left(X_{1}\right)=X_{1}+b X_{n}{ }^{q}$ for some nonnegative integer $q$. If $\sigma^{\prime}$ acts as the identity on $A^{\langle\sigma\rangle}$, then $\sigma^{\prime \prime}=\sigma^{s}$ for some $0 \leq s \leq p-1$, i.e. $b=s a$.

Proof. $A^{\langle\sigma\rangle}=k\left[U, X_{2}, \cdots, X_{n}\right]$ with $U=X_{1}{ }^{p}-a^{p-1} X_{n}{ }^{q(p-1)} X_{1}$, so $\sigma^{\prime}$ acts on $A^{\langle\sigma\rangle}$ as $\sigma^{\prime}\left(X_{j}\right)=X_{j}$ for $j>1$ and $\sigma^{\prime}(U)=U+\left(b^{p}-a^{p-1} b\right) X_{n}{ }^{p q+1}$. Thus $\sigma^{\prime}$ acts trivially on $A^{\langle\sigma\rangle}$ iff $b=0$ or $a^{p-1}=b^{p-1}$, i.e. $b=0$ or $b=\xi a$ for $\xi$ a $(p-1)$ th root of unity. Since the $(p-1)$ th roots of unity in $k$ are $1,2, \cdots$, $p-1$ the lemma follows.

Lemma 8. With the notation as in Lemma $6, \sigma_{i+1}$ acts nontrivially on $A^{K_{1 i}}$.

Proof. We will actually show that if $\sigma$ is an automorphism of $A$ having the form $\sigma\left(X_{1}\right)=X_{1}+a X_{n}$ and $\sigma\left(X_{j}\right)=X_{j}$ for $j>1$ which acts trivially on $A^{K_{1 i}}$, then $\sigma \in K_{1 i}$. Lemma 7 (with $q=0$ ) is just the case $i=1$ of this claim. Assume that the lemma is true for $i<q+1$. Consider an automorphism $\sigma$ having the above form whose restriction to $A^{K_{1, q+1}}$ is trivial. Look at both $\sigma$ and $\sigma_{q+1}$ as restricted to $A^{K_{1 q}}$.

Lemma 6 tells us that this ring is $k\left[U_{q}, X_{2}, \ldots, X_{n}\right]$ and that $\sigma\left(U_{q}\right)=U_{q}+$ $b X_{n}{ }^{p q}, \sigma_{q+1}\left(U_{q}\right)=U_{q}+c X_{n}{ }^{p q}$. $\sigma$ acts trivially on $A^{K_{1 q}}$, so Lemma 7 tells us that $\sigma=\sigma_{q+1} s$ when restricted to this ring. Thus $\sigma \sigma_{q+1}^{-s}$ is the identity on $A^{K} K_{1 q}$ and so $\sigma \sigma_{q+1}^{-s}$ is in $K_{1 q}$. Therefore, $\sigma$ is in $K_{1, q+1}$ and the lemma is proved. 
Corollary. $A^{K_{1}}=k\left[U_{1}, X_{2}, \cdots, X_{n}\right]$ where $U_{1}=\Sigma c_{q} X_{n}{ }^{p^{r}-p^{q}} X_{1}{ }^{p q}$ (the sum being from $q=0$ to $q=r$ ), with $c_{r}=1$ and $c_{0} \neq 0$.

For any $\sigma$ in $K^{\prime}$ but not in $K_{1}$ we note that $\sigma\left(U_{1}\right)=U_{1}$, so $K_{2} \oplus \cdots \oplus$ $K_{n-1}$ acts on $A^{K_{1}}$ in such a fashion that we can inductively compute $A^{K^{\prime}}$. The result is

Proposition 3. $A^{K^{\prime}}=k\left[U_{1}, \cdots, U_{n-1}, X_{n}\right]$ where $U_{i}=\Sigma c^{i}{ }_{q} X_{n}{ }^{p^{r}-p^{q}} X_{i}^{p^{q}}$ (the sum being from $q=0$ to $q=r$ ), with $c^{i}{ }_{r}=1$ and $c^{i}{ }_{0} \neq 0$.

Clearly $\tau\left(U_{i}\right)=U_{i}$, so $A^{K}=\left(A^{K}\right)^{\prime}\langle\tau\rangle=k\left[U_{1}, \cdots, U_{n-1}, X_{n}^{m}\right]$. As $A^{K}$ is a polynomial ring $\Omega_{A} K$ is a free module and so is naturally isomorphic to $\Omega^{z}{ }_{A} K=$ $A^{K}\left(d U_{1}, \cdots, d U_{n-1}{ }^{K}, d\left(X_{n}{ }^{m}\right)\right)$, i.e. the free $A^{K}$ module generated by $d U_{1}, \cdots$, $d U_{n-1}, d\left(X_{n}{ }^{m}\right)$. As $c^{i} \neq 0$ this is the $A^{K}$ submodule of $\Omega^{z}{ }_{A}$ generated by $X_{n}^{p^{r}-2} \omega_{1}, \cdots, X_{n}^{p^{r}-2} \omega_{n-1}, X_{n}^{m-1} d X_{n}$ where $\omega_{i}=X_{n} d X_{i}-X_{i} d X_{n}$.

Now we wish to compute $\left(\Omega^{z}{ }_{A}\right)^{K}$ and to do this we will compute $\left(\Omega^{z}{ }_{A}\right)^{K_{1}}$, $\left(\Omega_{A}^{z}\right)^{K}$ and finally $\left(\Omega^{z}{ }_{A}\right)^{K}$.

Let $\omega=\Sigma a_{i} d X_{i}$ be an element of $\left(\Omega^{z}{ }_{A}\right)^{K_{1}}$ and let $\sigma$ be an element of $K_{1}$. Then $\sigma(\omega)-\omega=\Sigma\left(\sigma\left(a_{i}\right)-a_{i}\right) d X_{i}+a_{\sigma} \sigma\left(a_{i}\right) d X_{n}=0$. (Here as before $\sigma\left(X_{1}\right)=$ $X_{1}+a_{\sigma} X_{n}$ and $\sigma\left(X_{j}\right)=X_{j}$ for $j>1$.) Thus $\sigma\left(a_{i}\right)=a_{i}$ for $i<n$ and $\sigma\left(a_{n}\right)-$ $a_{n}=-a_{\sigma} a_{1}$. But as $\sigma$ is ramified at $X_{n}, a_{1}$ must be of the form $a_{1}{ }^{\prime} X_{n}$ for some $a_{1}^{\prime}$ in $A^{K_{1}}$. So $\omega=a_{1}^{\prime}\left[X_{n} d X_{1}-X_{1} d X_{n}\right]+a_{2} d X_{2}+\cdots+a_{n-1} d X_{n-1}+$ $\left[a_{n}+a_{1}^{\prime} X_{1}\right] d X_{n}$, which is in $A^{K}{ }^{1}\left(\omega_{1}, d X_{2}, \cdots, d X_{n}\right)$, which in turn is containea in $\left(\Omega^{z}{ }_{A}\right)^{K_{1}}$. Thus $\left(\Omega^{z}\right)^{K_{1}}=A^{K_{1}}\left(\omega_{1}, d X_{2}, \cdots, d X_{n}\right)$.

Similiar computations show that $\left(\Omega_{A}^{z}\right)^{K^{\prime}}=A^{K^{\prime \prime}}\left(\omega_{1}, \cdots, \omega_{n-1}, d X_{n}\right)$.

Now $\left(\Omega^{z}\right)^{K^{\prime}}$ and $\left(\Omega^{z}{ }_{A}\right)^{(\tau)}$ are both subsets of $\Omega_{A}^{z}$ and their intersection is $\left(\Omega^{z}{ }_{A}\right)^{K}$. But $A^{(\tau)}=k\left[X_{1}, \cdots, X_{n-1}, X_{n}{ }^{m}\right]$, so $\left(\Omega^{z}{ }_{A}\right)^{\langle\tau\rangle}=\Omega_{A}^{z}(\tau)=$ $A^{\langle\tau\rangle}\left(d X_{1}, \cdots, d X_{n-1}, X_{n}^{m-1} d X_{n}\right)$. So if $\omega \in\left(\Omega^{z}{ }_{A}\right)^{K}$, then $\omega=b_{1} d X_{1}+\cdots+$ $b_{n-1} d X_{n-1}+b_{n} X_{n}^{m-1} d X_{n}$ with $b_{i} \in A^{\langle\tau\rangle}$, and also $\omega=a_{1} \omega_{1}+\cdots+a_{n-1} \omega_{n-1}$ $+a_{n} d X_{n}$ with $a_{i} \in A^{K^{\prime}}$. Comparing coefficients we see that $b_{i}=a_{i} X_{n}$ for $i<n$. But as $b_{i} \in A^{\langle\tau\rangle}$ the fact that $X_{n}$ divides $b_{i}$ implies $X_{n}{ }^{m}$ divides $b_{i}$ and so $a_{i}=a_{i}^{\prime} X_{n}{ }^{m-1}$. And as $a_{i} \in A^{K^{\prime}}$ it follows that $a_{i}^{\prime} \in A^{n} K^{\prime}$. Now $a_{n}=a_{1} X_{1}+\cdots+a_{n-1} X_{n-1}+b_{n} X_{n}^{m-1}=\left[a_{1}{ }^{\prime} X_{1}+\cdots+a_{n-1}{ }^{\prime} X_{n-1}+b_{n}\right] X_{n}^{m-1}$, or writing $a_{n}^{\prime}=\left[a_{1}^{\prime} X_{1}+\cdots+a_{n-1}{ }^{\prime} X_{n-1}+b_{n}\right], a_{n}=a_{n}{ }^{\prime} X_{n}{ }^{m-1}$; and here too we have $a_{n}{ }^{\prime} \in A^{K^{\prime}}$. Thus $\omega=\left[a_{1}{ }^{\prime} \omega_{1}+\cdots+a_{n-1}{ }^{\prime} \omega_{n-1}+a_{n}{ }^{\prime} d X_{n}\right] X_{n}{ }^{m-1}$, which is in $A^{K}\left(X_{n}^{m-1} \omega_{1}, \cdots, X_{n}{ }^{m-1} \omega_{n-1}, X_{n}{ }^{m-1} d X_{n}\right)$. Thus $\left(\Omega_{A}^{z}\right)^{K}=$ $A^{K}\left(X_{n}^{m-1} \omega_{1}, \cdots, X_{n}^{m-1} \omega_{n-1}, X_{n}^{m-1} d X_{n}\right)$.

Thus $\Omega^{z} \mu: \Omega_{A}^{z} \rightarrow_{(}\left(\Omega^{z}\right)^{K}$, always an inclusion, is not an epimorphism if $m<p^{r}-1$; for then $X_{n}{ }^{m-1} \omega_{1}$, for example, is not in its image. Thus, $\Omega^{z} \mu$ is an isomorphism iff $m=p^{r}-1$. 
Clearly $X_{n}{ }^{m-1} \omega_{i}$ and $X_{n}{ }^{m-1} d X_{n}$ are in $\left(\Omega_{A}^{z}\right)^{H}$, so $\left(\Omega_{A}^{z}\right)^{H}=$ $A^{H}\left(X_{n}^{m-1} \omega_{1}, \cdots, X_{n}{ }^{m-1} \omega_{n-1}, X_{n}{ }^{n-1} d X_{n}\right)$. So if $m<p^{r}-1$, then $X_{n}{ }^{m-1} \omega_{1}$, for example, is in $\left(\Omega^{z}{ }_{A}\right)^{H}$ but not in the image of $\Omega^{z} \beta$, i.e. $\Omega^{z} \beta$ is not surjective.

To complete the proof of Proposition 2 we must show that if $m=p^{r}-1$, then $\Omega^{z} \beta$ is an isomorphism iff $H=K$. As $\Omega^{z}{ }_{A}{ }=\left(\Omega_{A}^{z}\right)^{K}$ when $m=p^{r}-1$, the "if" part is done. Now suppose $H \neq K$, so that also $H^{\prime} \neq K^{\prime}$. Let $\sigma$ be an element of $H^{\prime}$ not in $K^{\prime}$ and let $H_{1}$ be the subgroup of $H$ generated by $K$ and $\sigma$. Note that the $p$ subgroup of $H_{1}$ is the subgroup of $H^{\prime}$ generated by $K^{\prime}$ and $\sigma$.

Lemma 9. $\sigma$ acts nontrivially on $A^{K}$.

Proof. If $\sigma$ acts trivially on $A^{K}$, then $\sigma$ acts trivially on $A^{K^{\prime}}$, and so it acts on $A^{K_{1} \oplus \cdots \oplus K_{n-2}}=k\left[U_{1}, \cdots, U_{n-2}, X_{n-1}, X_{n}\right]$ as $\sigma\left(U_{j}\right)=U_{j}, \sigma\left(X_{n-1}\right)=$ $X_{n-1}+a X_{n}, \sigma\left(X_{n}\right)=X_{n}$. As $\sigma$ acts trivially on $A^{K^{\prime}}$, Lemma 8-suitiably interpreted-shows that there is an element $\sigma_{n-1}$ of $K_{n-1}$ so that $\sigma \sigma_{n-1}{ }^{-1}$ acts as the identity on $A^{K_{1} \oplus \cdots \oplus K_{n-2}}$. Similiar such arguments-using Lemma 8 at each stage-show that there are $\sigma_{i} \in K_{i}$ so that $\sigma\left(\sigma_{1} \cdots \sigma_{n-1}\right)^{-1}$ acts as the identity on $A$. Thus $\sigma=\sigma_{1} \cdots \sigma_{n-1}$ and so is an element of $K^{\prime}$.

Thus by making a suitable change of coordinates we may assume that $\sigma$ acts on $A^{K^{\prime}}$ as $\sigma\left(U_{1}\right)=U_{1}+b X_{n}{ }^{p^{q}}$ with $b \neq 0, \sigma\left(U_{j}\right)=U_{j}$ for $j>1$, and $\sigma\left(X_{n}\right)=X_{n}$. From this we deduce that $A^{K}=\left(A^{K^{\prime}}\right)^{(\sigma)}=k\left[V_{1}, U_{2}, \cdots, U_{n-1}, X_{n}^{p^{r}-1}\right]$ where $V_{1}^{n}=U_{1}{ }^{p}-b X_{n}{ }^{p^{q}(p-1)} U_{1}$. Thus $A^{H_{1}}=k\left[V_{1}, U_{2}, \cdots, U_{n-1}^{n-1}, X_{n}^{p^{r}-1}\right]$, and so $\Omega^{z}{ }_{A} H_{1}=A^{H_{1}}\left(d V_{1}, d U_{2}, \cdots, d U_{n-1}, X_{n}{ }^{p^{r}-2} d X_{n}\right)$. Thus even if $m=$ $p^{r}-1$, if $H \neq K$, then $\Omega^{z} \beta$ is not surjective as, for example, $d U_{1}$ is not in the image of $\Omega^{z} \beta$ as it is not even in $\Omega^{z}{ }_{A} H_{1}$. This completes the proof of Proposition 2.

Proof of Theorem 1. Let $x$ be one of the points of codimension 1 passing through $x_{0}$, and let $H$ be its ramification group. If the order of $H$ is prime to $p$, then $\Omega^{z} \pi_{y}$ is an isomorphism by Theorem 3 of Chapter II.

If $H$ satisfies (b), then $H^{\prime}=H_{1} \oplus \cdots \oplus H_{t}$ where each $H_{i}$ is invariant under conjugation by $\tau$ and each has order $p^{r}$. From our earlier work we may assume that $H$ acts on $A=k\left[X_{1}, \cdots, X_{n}\right]$. As $\tau$ acts on $H_{i}$ by conjugation and has order $p^{r}-1$ while $H_{i}$ has order $p^{r}$, it follows that all elements of $H_{i}$ have the same type. But then, as remarked just before Lemma 2, the size of a maximal independent set in $H^{\prime}$ is no more than $t$. Now suppose $\left\{\sigma_{1}, \cdots, \sigma_{s}\right\}$ is a maximal independent set in $H^{\prime}$. Then by Lemma $3, H^{\prime}$ can be assumed to act trivially on $X_{s+1}, \cdots, X_{n}$. Therefore the decomposition ideal of $H$ contains $\left(X_{s+1}, \cdots, X_{n}\right)$ and so has height $\geq n-s$ and corank $\leq s$. But by assumption the corank of the decomposition ideal is $t$, therefore $t=s$. In fact if $\sigma_{i}$ is any 
nonidentity element from $H_{i}$, then $\left\{\sigma_{1}, \cdots, \sigma_{t}\right\}$ is a maximal independent subset of $H^{\prime}$.

Now, as established in the remarks immediately following Lemma 3, we may safely assume that $t=n-1$. Let $K, K^{\prime}$ and $K_{i}$ be as defined earlier in the text. $K_{i} \subset H_{i}$ and $m$ divides order $\left(K_{i}\right)-1$, and so $K_{i}=H_{i}$. But then $K^{\prime}=H^{\prime}$ and $K=H$. Proposition 2 now tells us that $\Omega^{z} \beta$ is an isomorphism, and so by our original reduction $\sigma^{z} \pi_{y}$ is also.

For the converse, note that either the order of $H$ is prime to $p$ (condition (a)) or not. If not, but $\Omega^{z} \pi_{y}$ is an isomorphism, then $\Omega^{z} \beta$ is also an isomorphism. Let $\left\{\sigma_{1}, \cdots, \sigma_{t}\right\}$ be a maximal independent subset of $H^{\prime}$. Form $K, K^{\prime}$ and the $K_{i}$ as in the text. Considering $H$ as acting on the local ring $A_{\left(X_{1}, \cdots, X_{n}\right)}$, the decomposition ideal will be the extension of $\left(X_{t+1}, \cdots, X_{n}\right)$, and this has corank $t$. But the morphism from $\mathcal{O}_{0}$ to $\mathcal{O}$ established in the first reduction at the beginning of this chapter induces a bijection between the prime ideals of the two rings, and so the corank of the decomposition ideal of $H$ acting on $\mathcal{O}$ is also $t$. Again the remarks following Lemma 3 tell us that we may assume that $t=n-1$. But then by Proposition 2, $H=K, H^{\prime}=K^{\prime}=K_{1} \oplus \cdots \oplus K_{t}$, each $K_{i}$ has order $p^{r}$ and $K / K^{\prime}$ has order $p^{r}-1$. Simply putting $H_{i}=K_{i}$ gives condition (b).

\section{CHAPTER IV. EXAMPLES AND COMPLEMENTS}

The theorem of Chapter III does settle one important case affirmatively. (Keep in mind that the base field is algebraically closed.)

Proposition 1. Let $X$ be an arbitrary nonsingular variety. Let $G$ be the r-fold symmetric group acting on $X^{r}$ by permuting the factors, so that $X^{r} / G$ is the r-fold symmetric product of $X$. Then $\Omega^{z} \pi: \Omega_{X^{r / G}}^{z} \rightarrow\left(\pi_{*} \Omega_{X^{r}}^{z}\right)^{G}$ is an isomorphism.

Proof. If the dimension of $X$ is greater than 1 or if the characteristic of $k$ is not 2 , then $\pi$ is tamely ramified at each point of codimension 1 . If the dimension of $X$ is 1 and the characteristic of $K$ is 2, then the inertia group of a point of codimension 1 is $\mathrm{Z} / 2 \mathrm{Z}$ and, as $G$ does act linearly, condition (b) of the theorem is satisfied.

Remark. This proposition holds equally well if any subgroup of the symmetric group is used for $G$, as the inertia group of a point of codimension 1 will be either the identity of $\mathrm{Z} / 2 \mathrm{Z}$.

Another case which is rather easily settled is when a group $G$ acts linearly on a 2-dimensional variety. For simplicity suppose the characteristic of $k$ is $p \neq 0$, and suppose $G$ is a linear group of automorphisms of $\mathrm{A}^{2}$, which is the ramification group of a point of codimension 1 . As $G$ can contain only one type 
of automorphism, the order of $G$ is $m p^{r}$ where $m$ divides $p^{r}-1$. In this case Theorem 1 of Chapter III tells us that $\Omega^{z} \pi$ is an is omorphism iff the order of $G$ is prime to $p$ or else is $\left(p^{r}-1\right) p^{r}$ for some $r>0$. Thus in the case of dimension 2 we have an isomorphism iff the inertia group at every point of codimension 1 has the "right" order.

Now suppose $G$ is a linear group of automorphisms of $\mathrm{A}^{n}, n>2$, which is the ramification group at a point of codimension 1. If the order of $G$ is divisible by $p$, but $\Omega^{z} \pi$ is an isomorphism, then the order of $G$ is $\left(p^{r}-1\right) p^{r s}$ with $s<n$. Unlike the case $n=2$, the order of $G$ being "right" is not enough to insure that $\Omega^{z} \pi$ is an isomorphism. In fact with $k$ algebraically closed, for any positive integer $r$, any $n>2$ and any $1<s<n$ there are groups $G$ and $H$ of order $\left(p^{r}-1\right) p^{r s}$ which act linearly on $\mathbf{A}^{n}$, are ramified at a unique point of codimension 1 and are the ramification groups of that point, so that for $G$ the map of Zariski differentials is an isomorphism, but for $H$ it is not.

Example 1. Let $A=k\left[X_{1}, \cdots, X_{n}\right]$ with $n>1$. Let $r$ be any positive integer and $0<s<n$. Let $G$ be the group of linear automorphisms of $A$ generated by $\sigma_{i}\left(X_{i}\right)=X_{i}+X_{n}$ for $1 \leq i \leq s, \sigma_{i}\left(X_{j}\right)=X_{j}$ for $j \neq i ; \tau\left(X_{j}\right)=X_{j}$ for $j<n$, $r\left(X_{n}\right)^{i}=\xi X_{n}$ with $\xi$ a primitive $p^{r}-1$ root of unity. In this case $G$ is ramified at $\left(X_{n}\right), G$ is the ramification group, and the decomposition ideal has corank $s$. Putting $G_{i}$ equal to the smallest subgroup of $G$ which contains $\sigma_{i}$ and is closed under conjugation by $\tau$, we see that $G^{\prime}=G_{1} \oplus \ldots \oplus G_{s}$ and that each of these has order $p^{r}$. Thus in this case the quotient map induces an isomorphism for the Zariski differentials.

Example 2. Let $A, n, r$ and $s$ be as in Example 1, except assume that $s>1$. Let $H$ be the group of linear automorphisms generated by $\sigma_{i}\left(X_{1}\right)=X_{1}+$ $\xi X_{n}$ with $\xi_{i}$ a primitive $p^{i}-1$ root of unity for $1 \leq i \leq s, \sigma_{i}\left(X_{j}\right)=X_{j}$ for $j>1$; $\tau\left(X_{j}\right)=X_{j}$ for $j<n, \tau\left(X_{n}\right)=\xi X_{n}$ with $\xi$ a primitive $p^{r}-1$ root of unity. $H$ is ramified at $\left(X_{n}\right)$ and $H$ is the ramification group. $H$ has order $\left(p^{r}-1\right) p^{r s}$ as desired, but $H^{\prime}$ has elements of only one type, while the corank of the decomposition ideal is $n-1$. Thus in this case $\Omega^{z} \pi$ is not an isomorphism.

Remark. If, in Example 1, $m m^{\prime}=p^{r}-1$, then by considering instead of $G$ the group generated by $\tau^{m}$ and the $\sigma^{\prime}$ 's we will get a group of order $m p^{r s}$ with $m<p^{r}-1$ so that the condition " $m=p^{r}-1$ " in part (b) of the theorem is not redundant.

If $H$ is the ramification group of some point of codimension 1 on $\mathrm{A}^{n}$ and $H$ acts linearly, then of necessity the order of $H$ is $m p^{r}$ with $m$ dividing $p^{r}-1$. While the order of $H$ does not-except when $n=2-$ tell us if $\Omega^{z} \pi$ is an isomorphism, it does go a fair way. If the action is not linear, then even if $n=2$ the order seems to tell us nearly nothing. In particular the order of $H$ will be $m p^{r}$ with $m$ prime to $p$, but $m$ need not divide $p^{r}-1$. 
Example 3. Let $A=k[X, Y]$ with the characteristic of $k=2$. Let $H$ be generated by $\sigma(X)=X, \sigma(Y)=Y+X^{3} ; \tau(X)=\xi X, \tau(Y)=Y$ with $\xi$ a primitive cube root of unity. Note that $\sigma \tau=\tau \sigma$ so that $H$ is the cyclic group of order 6 . Thus $m=3, p=2$ and $r=1$ so that $m$ does not divide $p^{r}-1$.

By computations similar to our earlier ones we find that $A^{H}=$ $k\left[X^{3}, Y\left(Y+X^{3}\right)\right], \Omega^{z}{ }_{A}=A^{H}\left(X^{2} d X, X^{2}(Y d X+X d Y)\right)$ and $\left(\Omega_{A}^{z}\right)^{H}=$ $A^{H}\left(X^{2} d X, X^{2}(Y d X+X d Y)\right)$. So in this case the map $\Omega^{z} \pi$ is an isomorphism.

Example 4. Let $A=k[X, Y]$ with the characteristic of $k=2$. Let $H$ be the cyclic group of order 2 with generator $\sigma$ acting by $\sigma(X)=X$ and $\sigma(Y)=Y+$ $X^{2}$. If this were a linear action, then we would have an isomorphism of Zariski differentials. But here $A^{H}=k\left[X, Y\left(Y+X^{2}\right)\right]$ so that $\Omega^{z}{ }_{A}{ }^{H}=A^{H}\left(d X, X^{2} d Y\right)$ while $\left(\Omega^{z}{ }_{A}\right)^{H}=A^{H}(d X, d Y)$. Thus the two modules are not isomorphic.

In general the situation for nonlinear actions, even on affine space, seems very different. Results of computations have led me to feel that isomorphisms are very rare and "accidental".

\section{REFERENCES}

1. R. E. Briney, Intersection theory on quotients of algebraic varieties, Amer. J. Math. 84 (1962), 217-238. MR 25 \#3935.

2. A. Grothendieck, Revêtements étales et groupe fondamental (Séminaire de géométrie algébrique du Bois Marie 1960/61), Lecture Notes in Math., vol. 224, SpringerVerlag, Berlin and New York, 1971.

3, - Éléments de géométrie algébrique. IV, Inst. Hautes Études Sci. Publ. Math. No. 32 (1967), 361 pp. MR $39 \# 220$.

4. J. Lipman, Free derivation modules on algebraic varieties, Amer. J. Math. 87 (1965), 874-898. MR $32 \# 4130$.

5. A. Mattuck, Symmetric products and Jacobians, Amer. J. Math. 83 (1961), 189206. MR $26 \# 122$.

6. - On symmetric products of curves, Proc. Amer. Math. Soc. 13 (1962), 8287. MR $25 \# 76$.

7. A. Mattuck and A. Mayer, The Riemann-Roch theorem for algebraic curves, Ann. Scuola Norm. Sup. Pisa (3) 17 (1963), 223-237. MR $29 \# 102$.

8. P. Samuel, Anneaux gradués factoriels et modules réflexifs, Bull. Soc. Math. France 92 (1964), 237-249. MR $32 \# 4160$.

9. J. P. Serre, Graupes algébriques et corps de classes, Actualités Sci. Indust., no. 1264, Hermann, Paris, 1959. MR 21 \#1973; errata, MR 30 p. 1200.

10. S. Suzuki, On torsion of the module of differentials of a locality which is a complete intersection, J. Math. Kyoto Univ. 4 (1964/65), 471-475. MR 31 \#4809.

11. O. Zariski, An introduction to the theory of algebraic surfaces, Lecture Notes in Math., no. 83, Springer-Verlag, Berlin and New York, 1969. MR 41 \#8418.

12. O. Zariski and P. Samuel, Commutative algebra. Vol. I, University Series in Higher Math., Van Nostrand, Princeton, N. J., 1957. MR 19, 833.

DEPARTMENT OF MATHEMATICS, UNIVERSITY OF PUERTO RICO, RIO PIEDRAS, PUERTO RICO 